\title{
An Economic Analysis of Disaggregation of Space Assets: Application to GPS
}

\author{
Daniel E. Hastings ${ }^{\mathrm{a} 1}$, Paul A. La Tour ${ }^{\mathrm{b} 2}$ \\ ${ }^{\mathrm{a}}$ Department of Aeronautics and Astronautics \\ ${ }^{b}$ Institute for Data, Systems \& Society, MIT, Cambridge, MA 02139
}

New ideas, technologies and architectural concepts are emerging with the potential to reshape the space enterprise. One of those new architectural concepts is the idea that rather than aggregating payloads onto large very high performance buses, space architectures should be disaggregated with smaller numbers of payloads (as small as one) per bus and the space capabilities spread across a correspondingly larger number of systems. The primary rationale is increased survivability and resilience. The concept of disaggregation is examined from an acquisition cost perspective. A mixed system dynamics and trade space exploration model is developed to look at long-term trends in the space acquisition business. The model is used to examine the question of how different disaggregated GPS architectures compare in cost to the well-known current GPS architecture. A generation-over-generation examination of policy choices is made possible through the application of soft systems modeling of experience and learning effects. The assumptions that are allowed to vary are: design lives, production quantities, nonrecurring engineering and time between generations. The model shows that there is always a premium in the first generation to be paid to disaggregate the GPS payloads. However, it is

\footnotetext{
${ }^{1}$ Professor, 33-213, MIT, AIAA Fellow

${ }^{2}$ PhD student, E38-520, MIT
} 
possible to construct survivable architectures where the premium after two generations is relatively low.

\section{Introduction}

Since the dawn of the space age nations have developed many important space based capabilities that are now further enhanced by operating inside larger architectural concepts. Thus the development of satellite constellations enabled new capabilities from space. A satellite constellation is defined[1] as a "distribution of satellites of similar types or with similar functions in similar or complementary orbits to accomplish a specific task under shared control". These constellations include essential capabilities such as global communications with geosynchronous (GEO) satellites, precision navigation and timing anywhere on the surface of the globe and weather monitoring from satellites to enable accurate forecasts. Over time these satellites have consistently become more massive, powerful and longer lived. For example, many GEO communications satellites launched during the last decade weighed around 2,000 kg, operated near 10,000 W of power, and will last at least 12 years[2]. These are much larger and more powerful than the earliest GEO communications satellites that had only a few $\mathrm{kW}$ of power and lasted for a small number of years. This improvement is due to continuous advances in technology such as the technology of solar arrays and the use of more advanced propulsion engines with much higher specific impulses.

These large, powerful, long-lasting space vehicles have largely become the norm for bigticket space projects. However, architecture designs are increasingly lagging behind the rapid development of new technologies. It is often seen that architectures designed for the economic and technological realities at the time of launch are not adequate for the actual present-day environment[2]. These large monolithic space vehicles-while highly sophisticated and reliablehave become characterized by increasing costs and lengthening development timelines, in addition to increasing launch and insurance costs. Furthermore, long design lifetimes may contribute to the obsolescence of onboard technology and the under-utilization of increasingly rapid technological 
advances. Another recent example are the GPS satellites which have gone from a 7.5 year design lifespan to a 15 year design lifespan[3]. If they use technology that becomes obsolete on five-year timescales then a fifteen-year old satellite will be three generations behind by the end of its life.

This raises questions about the long-term viability of current space architecture design practices. One possible change to the existing design practice is by implementing smaller, more responsive constellations of spacecraft which could take advantage of increased miniaturization and faster advancement in technology[4]. Such systems would have short design lives and would therefore be more in sync with current technological change rates.

There are a number of other new ideas emerging in the business of designing new space systems. Some of these are inspired by the Cubesat revolution[5] which is bringing fast, technologically up to date capabilities to the space enterprise, albeit the low earth orbit space enterprise. Other concepts are emerging such as public-private partnerships[6] in space, commercial space launch to low earth orbit as well as older ideas such as on-orbit servicing[7]. All of these ideas promise to reshape parts of the dominant space architecture paradigm.

One of the concepts introduced within the last two decades has been the idea of distributed satellite systems (DSS)[8] where the mission or function of the space system is required to be spread across a number of satellites (not necessarily homogenous ones) in order for the function to be realized. Note that while some authors[9] explicitly identified DSS with constellations of small satellites, this is not required by the definition. By the definition, GPS is a DSS since the function of precision navigation and timing requires the acquisition of four signals from four separate satellites that are separated and the precision navigation cannot be realized from a single satellite. Furthermore, one would not describe GPS as a constellation of small satellites. Another example is Iridium[10] which requires more than one satellite in LEO in order for a user to make a satellite based phone call to anywhere on the globe. Yet another example is the GRACE mission[11] which 
needs two physically separated satellites which fly in formation in order to map the Earth's gravity field.

A number of other architectural concepts have emerged in the recent years. These are satellite swarms, formations, clusters, satellite disaggregation, fractionation and federation. The use of some of these terms is muddied by the fact that there are spacecraft missions named after some of them (thus, ESA has a mission called SWARM and one called CLUSTER). A satellite swarm is defined as a DSS consisting of many identical, low cost satellites autonomously cooperating to achieve a common goal[12]. A satellite formation is defined as multiple satellites working together in a group to achieve the mission of a larger satellite. A good example was the Techsat21 mission[13] which was planned to be a formation of small radar satellites which would together synthesize a larger space based radar in LEO. A satellite cluster refers to a group of formation flying satellites close together moving in almost identical orbits. Note that some of these definitions have significant overlap.

The concept of space disaggregation is defined[14] as "The dispersion of space-based missions, functions or sensors across multiple systems spanning one or more orbital plane, platform, host or domain". Note that this definition overlaps the DSS one. The difference is one of intent. A DSS must be distributed in order to fulfill the mission that it was intended to deliver. By contrast, disaggregation is "one strategy to improve resiliency" [14] where resiliency is defined as "Resiliency is the ability of a system architecture to continue providing required capabilities in the face of system failures, environmental challenges or adversary challenges". Therefore an aggregated system can accomplish a mission indeed several missions but of it is disaggregated then it is also a distributed system. An example of an aggregated mission but not a distributed one is GEOS-R[15] which is a GEO based weather satellite that provides high spatial resolution weather pictures of the Unites States, real time mapping of lightning and monitoring of solar activity. If the various instruments were distributed to different buses then they would be a disaggregated system. One way to consider the 
difference between distribution and disaggregation is to note that a disaggregated architecture is one where if it were re-aggregated would still accomplish a mission whereas physically putting together a distributed architecture would not accomplish a mission.

Yet another relatively new architectural concept is that of a federated satellite system. A federated satellite system (FSS)[16] is composed of heterogeneous spacecraft that opportunistically share resources. The resource sharing is enabled in the design but not always active. Typical resources that may be shared in a network of spacecraft are data, downlink bandwidth, computer storage and processing. This is enabled by the right kind of communication links. The definition allows sharing of resources such as power, that would have to be enabled by the right kind of emitters and receivers on the spacecraft and potentially also mass for example, fuel. Federated Satellite Systems are a class of distributed satellite systems.

There have been a number of studies showing how to optimize system architectures for various classes of distributed space systems[17]. The classical GPS is a distributed system but not a disaggregated system since each GPS satellite has several payloads. Another concept, fractionation, has emerged in recent years. In fractionation some of the elements of the bus or payload of a satellite are split out as free flyers that interact through wireless links with each other. It is conceptually similar to how a computer network operates. This concept introduces the value of the flexibility enabled by being able to replace just the CPU on a satellite (typically the part which becomes obsolete the fastest) and keep the solar arrays (which have a much longer technology obsolescence timescale). The value of flexibility has been explored for space systems and has been shown to lead to potentially different design choices[18]. There are no examples of operational space systems that have successfully implemented fractionation. By this definition, a fractionated system is a disaggregated one.

In the Air Force white paper[14] which introduced disaggregation, five approaches to achieving disaggregation are posited. These are "Fractionation, Functional Disaggregation, Hosted 
Payloads, Multi-Orbit and Multi-Domain". Functional Disaggregation means that sensors that were on a single bus are dispersed to separate buses. Hosted Payloads are a class of functional disaggregation where the primary payload has an independent mission and a secondary payload is added to it. For example, adding a space weather sensor to a communications satellite. Multi-Orbit disaggregation puts the disaggregated payloads on multiple orbital planes for example, at GEO and in LEO. Multi-Domain disaggregation would combine sensors from space with air and ground based systems. By these definitions, this work will concentrate on functional disaggregation.

This concept of (functional) disaggregation [14] is an architectural choice which would make complex space architectures more survivable and resilient. The concept has an intuitive attraction. If some space disaster such as a meteorite shower or a coronal mass ejection from the Sun were to strike a set of satellites performing an important function, then the more satellites over which the function is spread then it is more likely that some of them will survive, theoretically increasing the systems resiliency. However, this carries with it many assumptions, namely that that the potential satellites failures are all uncorrelated and that the space architecture can still perform with "holes" in it. One clear example where this might not be true is if satellite failure is due to a hypervelocity impact from a meteorite that then creates orbital debris that affects other satellites in nearby orbits. In this case, the failures would be correlated. However disaggregation offers some other advantages. Since it replaces monolithic very complex, multi function satellites with groups of simpler satellites, it offers the potential to reduce total acquisition cost from the economies of scale that come from manufacturing many of the same type of system. As long as the lifetimes of the disaggregated systems are shorter than the current systems, the concept of (functional) disaggregation also offers leverage in being better aligned with the pace of technology, helping to sustain the space industrial base and providing less opportunity for requirements to spiral out of control. All of these desirable attributes indicate that there should be some in-depth analytic treatment of the concept of disaggregation, to ascertain which of the claims made about this space architectural concept can actually deliver. 
There have been a number of recent bodies of work that have looked at disaggregation. A study by Thompson et al.[19] outlined model based conceptual design optimization methods and applied them to a specific class of space system design. They undertook a design of a disaggregated space based weather constellation and compared it to the Defense Weather Satellite System[20]. They found a substantial lifecycle cost saving compared to the large multifunction approach. This suggests that under some optimal circumstances, disaggregation may result in saving resources. This is very desirable. In contrast, another recent work explored some aspects of disaggregation for the space based weather system[21]. It accounted for the greater complexity associated with disaggregated systems (for example, the greater number of interfaces but not the learning associated with building many of the same system) and concluded that such systems (for the weather case) were more expensive. Thus they came to the opposite conclusion about disaggregation from Thompson et al. It must be noted that these previous works operated in a static timeframe considering only the construction of one disaggregated generation at a single point in time based on the context of the day. Thus, it is fair to say the case for and against disaggregation in space systems remains to be developed. What is needed is a methodology to look at disaggregation performing over many generations to see whether the benefits of building many systems with technology refresh and an updated space industrial base outweigh the costs of building, launching and operating many more systems.

In this paper, we develop such a general methodology to address the issues with different space architectural strategies over long periods of time. Existing cost and performance models optimize at a single point in time. This has the potential for myopic decisionmaking; the best option at every single point in time can lead to a sub-optimal design over multiple generations. For example, disaggregation in the first generation (using existing cost/performance models to optimize at a single point in time) always indicates a worse cost vs. performance solution than the existing design. This work looks beyond one generation to see if concepts of learning, experience and efficiency ever overcome the extra costs in the initial generations of disaggregation. We apply 
this methodology specifically to the question of functional disaggregation for the GPS system. The GPS system has the advantage of a large amount of publically available historical data and it is conceptually simpler than the complex space based weather system. The space based weather system has a significant number of complicated sensors that are different from each other. It thus allows for the possibility of different optimal architectures which may account for the different conclusions from Thomson[20] and Dwyer[21]. We seek to answer whether a disaggregated GPS can be made more resilient and have similar or reduced costs relative to a business as usual case. While GPS is used as an relevant example since there is a lot of publically available data on the current system and the choices that went into the current system [3], the methodology is not specific to GPS and can be used to answer a range of other questions on future architectural choices in space across multiple generations of acquisitions.

The rest of the paper has the following structure. Section II discusses the development of a methodology to combine system dynamics and tradespace exploration to address the issues with disaggregation. Section III applies the model to GPS and examines the consequences of differing levels of disaggregation for GPS. Section IV concludes the paper with a review of the results and identifies future work.

\section{Development of the Methodology}

With over forty years of experience in space development many cost and performance models exist for the evaluation and design of space assets[22]. According to Saleh, the designs of space systems have nearly unanimously converged at massive, complex, and long-lasting spacecraft with one key driver of this trend being the minimization of the cost-per-operational day[23]. Vehicles at this design point are more costly to build and require increasingly long timelines to design and build. In addition, they are associated with a strong aversion to the use of new methods or technologies (which might be less expensive or shorten production time) due to the risk involved in fielding firstof-a-kind systems. Some authors have used the term of "vicious cycles" to describe the current state 
of the industry; Wertz believes the space industry to be caught in a spiral, where higher costs lead to longer schedules and fewer missions, which lead to a demand for higher reliability and longer design lifetimes, which then lead back to higher costs[22]. Rising costs and increasing time may also be, ironically, associated with an industry focus on minimization of total life-cycle cost, as myopically lengthening the design life of systems may work in the near term to lower life-cycle cost, but hurt the evolution of systems over several decades.

In order to answer the questions about the effect of different architectural strategies (e.g., disaggregation) it is necessary to have a predictive model that will allow comparison of various acquisition strategies over time for space systems. Tradespace Exploration (TSE)[24] is good at predictively modeling cost and performance of candidate systems at a specific point in time under a specific set of assumptions or context variables. It, however, offers no direct means to evaluate the system under examination across time, other than making abstract assumptions about future world conditions set through the means of exogenous context parameters and re-computing a utility function (when this is done, it is called Epoch-Era analysis[25]). Thus, it specifically natively lacks the ability to predictively model changes over time.

System Dynamics (SD)[26] provides a high level of abstraction for investigation of complex problems over time and offers techniques for testing the impact of policy changes on systems. But its greatest strength, that is high-level abstraction, has the shortcoming of being unable to validate solutions against real world physics and cost models. While a SD model descriptively models overall trends, discrete points cannot be examined within the context of those trends with current tools.

In this work a method is developed to merge System Dynamics techniques with Tradespace Exploration techniques to create meaningful models and accurate predictions for space system architectures. This work builds on the SD application to on-orbit servicing of space systems[7]. Elements of this approach were described in a paper presented at the IAC 2016[27]. 
In order to implement the combination of SD and TSE, the six steps in Table 1 were followed in this section:

Table 1 STEPS \& ACTIONS in combining SD \& TSE

STEP ACTION

1. Define system boundary and Level of Analysis

2. Create System Dynamics Model for time period to be evaluated

3. Construct point in time Tradespace

Exploration a. What is exogenous and outside of model boundary

b. What is endogenous and can be represented by trends over time

c. What is endogenous and can be controlled by decision makers a. Causal loop diagramming based on literature

b. Simulate deductive model

c. Tune to inductive model

d. Determine "Context Variables" and implementation a. Construct Tradespace with design variables to produce design vector

b. Evaluate performance model(s)

c. Select appropriate cost model(s) 
d. Construct utility function for satellite constellation performance

4. Link Tradespace Exploration With System Dynamics

5. Construct and Evaluate Combined Utility

Function

6. Execute Evolutionary Planning on Desired Design Vector a. Determine loops that pass through

Tradespace Exploration Module

b. Determine impact of stocks on

Tradespace Exploration Cost or

Performance Modules

a. Consider end utility metrics vs average utility metrics over time

b. Weighting of satellite constellation performance vs industry performance

c. Performance expectations change over time, as such utility may change as well

a. Re-run simulation varying context curves

b. Re-Run simulation varying tuning parameters 
The integration of Systems Dynamics and Tradespace Exploration is a deterministic modeling approach. It does not preclude the inclusion of some stochastic elements, specifically failure of satellites, failure of launch vehicles, and change in performance under random perturbations; however, all calculations in this work are deterministic and this method does not seek to evaluate random events. The model is also primarily dynamic and continuous, though it does allow discrete events (e.g., in the block purchase of satellites which as will be seen as input pulses, or discrete levels in context variables).

This approach of combining SD and TSE offers one other unique capability. Because the evolution of a specific system is tied to the evolution of the industry that produces it, this approach can model the interaction between industry as a whole as well as the impact of a single program on the industry over time. This capability arises from modeling causal interaction between an industry's trends and the effect on a specific program, while accounting for time delay and the reality that the space industry is composed of multiple programs. The system boundary of the Space Industrial Base can be modeled in System Dynamics, while the specific architecture under examination can be modeled within Tradespace Exploration and System Dynamics. This effectively enables modeling the entire Space Industrial Base in a lower fidelity model of only System Dynamics, while simultaneously simulating changes over time with a specific satellite constellation evolution, as a higher fidelity model using System Dynamics and Tradespace Exploration.

From the standpoint of the system dynamists this approach uses Tradespace Exploration as a way to size discrete inputs into a production pipeline; in this case the ordering of satellites. From the perspective of the user employing TSE, System Dynamics serves as a way to determine the exogenous levels and inputs required for the determination of what constitutes "optimal."

\subsection{Defining a System Boundary}


For the purpose of illustrating the merged method this paper implements an abstraction of the acquisition and development of space systems as represented in Figure 1. This figure shows that at the highest level Industry as a whole continues to evolve and develop technology and processes regardless of what happens inside the Space Industry. Some of the technologies developed in the broader Industry may be used by space systems, e.g., solar cells, software such as communications protocols, and even processes such as test and evaluation. The Space Industry is a subset of industry as a whole, producing technologies specific to the Space Industry such as: rockets, satellite busses or ground control stations. These are systems or technologies that will not evolve outside the Space Industry.

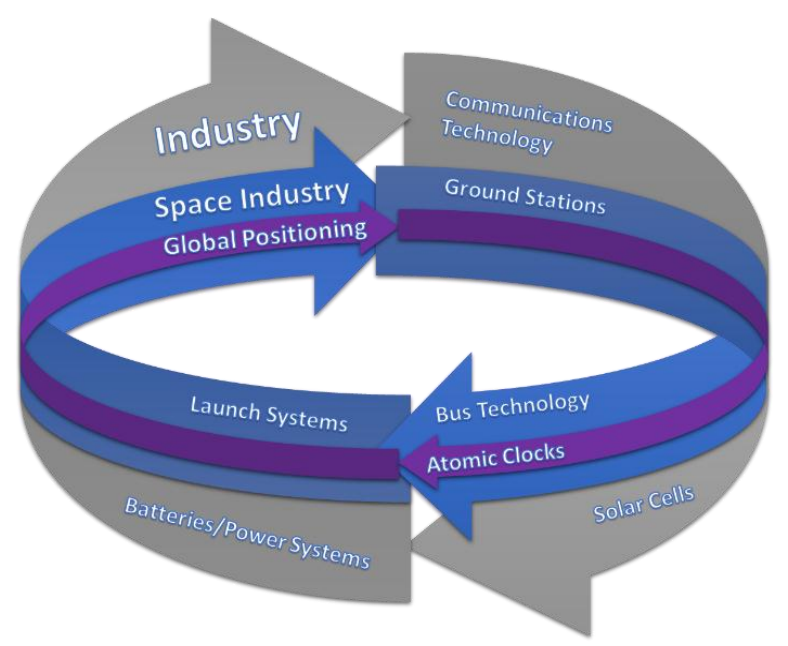

Figure 1 Model Representation of Space Acquisition \& Development

Within the Space Industry there are several different ways to functionally decompose activity. The tested merged model implements a worldview decomposition by functional area. Figure 1 breaks out one specific component of the Space Industry, the Global Positioning System (GPS). GPS makes use of technologies specific to the Space Industry but also develops capability that will be only used on precision navigation \& timing (PNT) programs, such as atomic clocks. However, some lessons learned and technology developed within the GPS sector of the Space Industry may be 
beneficial to other elements of the Space Industry and by extension Industry as a whole leading back to growth in the Space Industry.

GPS is a good illustrative example in explaining this mixed methodology, as it is a welldocumented system with a robust history and a well-defined set of services provided[3]. As Galileo[28], the European implementation of GPS, is a complementary system that offers the same type of service at a different design point comparing the evolution of both systems serves as a good test of this mixed methodology. This will be discussed later.

While the Space Industry includes many diverse actors and stakeholders, from civil agencies to the military to commercial entities, this analysis seeks to model industry-wide trends and their impact on satellite acquisitions. Trends in spacecraft design are not an issue affecting a single firm or entity, but rather an industry-wide phenomenon with ramifications for all stakeholders. The predictive model developed for this analysis will therefore focus primarily on the construction and upkeep of satellites, and track health over time. Activities in the overall space industry are exogenous to the model structure, and in theory the construction of individual satellites are below the level of analysis. Excluding satellites and their subcomponents, however, is problematic because the design of individual satellites impacts the Space Industry. To combat this level of analysis discrepancy the merged method offers its two-tiered modeling approach. Both the Space Industry and individual satellites are modeled together, so that a high-fidelity model operates within a lowfidelity model to capture the salient effects of both individual satellites and overall Space Industry trends. In Figure 1 the blue loop of Space Industry will be a lower level or more highly abstracted model, while the purple loop of a specific constellation will run at a higher level of fidelity with more detail. A System Dynamics model will capture the blue loop of the Space Industry. The purple loop of the GPS constellation will make use of Tradespace Exploration techniques for replenishment purposes as well as being modeled within the same System Dynamics model as the Space Industry as a whole. 


\subsection{Creating a System Dynamics Model}

\section{Causal Loop Diagramming}

It was noted previously that Wertz believes the Space Industry to be caught in a spiral, where higher costs lead to longer schedules and fewer missions, which lead to a demand for higher reliability and longer design lifetimes, which then lead back to higher costs[29]. This statement can be expressed as a causal loop diagram. (See Figure 2) Wertz specifically calls out the variables of cost, design schedule, number of missions, demand, reliability, and design life. This figure clearly displays a relationship among this set of variables. The diagram also notes that it is not just one loop that is active as Wertz claims, but in fact, four loops.

Figure 2 Wertz Loop in a Casual Loop Diagram

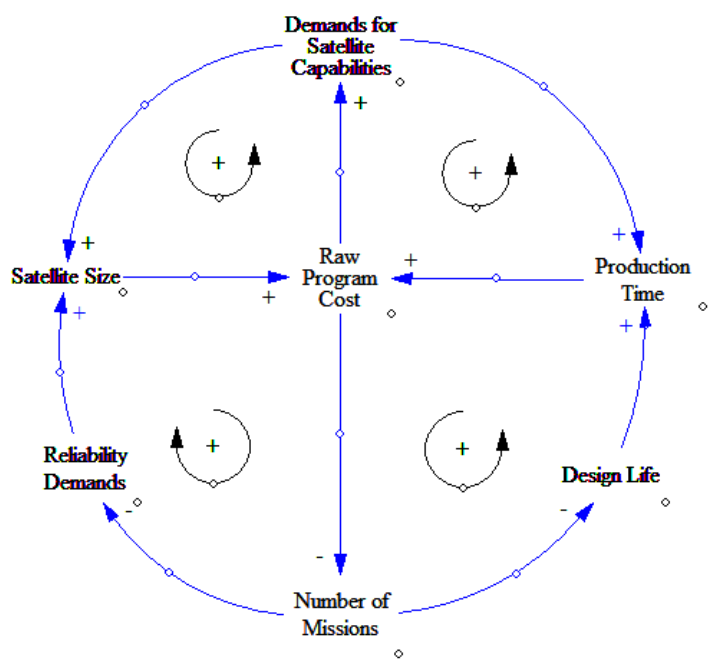


Saleh concludes that one key driver of the Space Industry is the minimization of the costper-operational day[23] We can integrate this concept with the previous loop as shown in Figure 3.

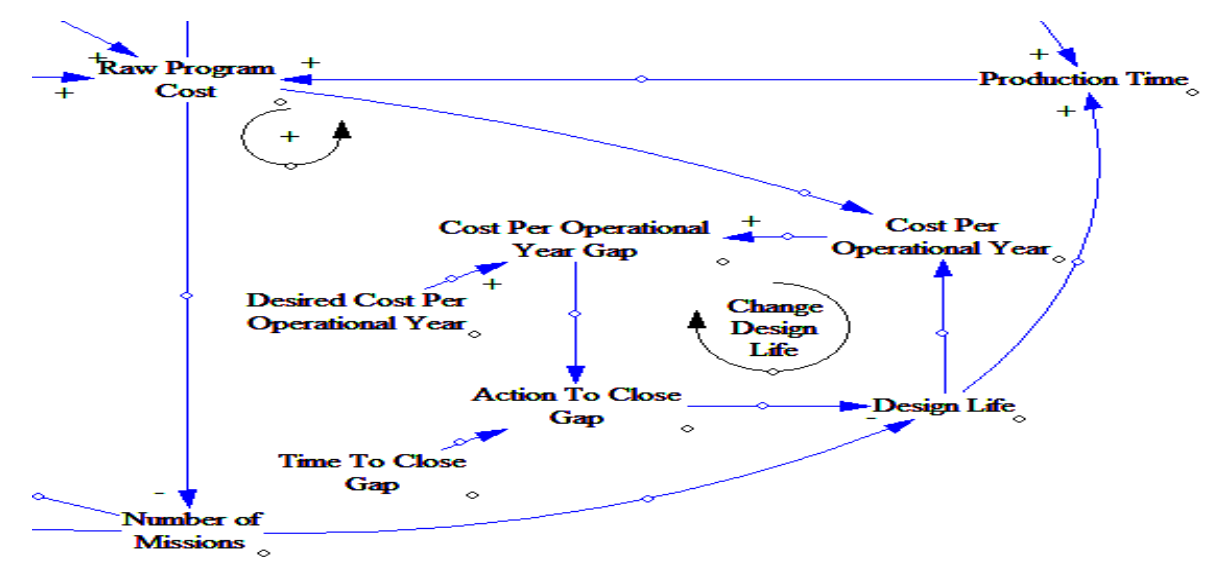

Figure 3 Saleh's Concept of cost per Operational Year

If Cost Per Operational Year is defined as Raw Program Cost divided by design life, we can see that how long a program lasts impacts its ability to meet the goal for operational cost per year. 
Concepts can be added to this causal loop diagram until all the major elements in the Space Industry have been abstracted by variables and connecting lines representing the direction of interaction. Each of these inclusions improves the causal loop diagram's ability to depict the realworld system. It is important to note that this causal loop diagram technique represents the system as defined in the literature, not as one would like it to be designed. Future policy recommendations can come from proposing changes to such a model once it is validated.

\section{Model Simulation}

Figure 4 presents how a number of structures have been pulled together and adapted for use in representing part of the Space Industry. The variable "Generate Request for Satellites (SV)" when pulsed creates a spike in production similar to the effect of signing a contract. While this is not a perfect abstraction of what happens in the real world, both "SV production" and "SVs in Production" represent third-order material delays, and thus provide a reasonable smoothing effect in producing and launching satellites. On the basis of the design life produced, the satellites will decay on orbit until they run out. Similarly, on the basis of the production time required the model will trigger a new acquisition to ensure the required capability on orbit does not fall below the desired level.

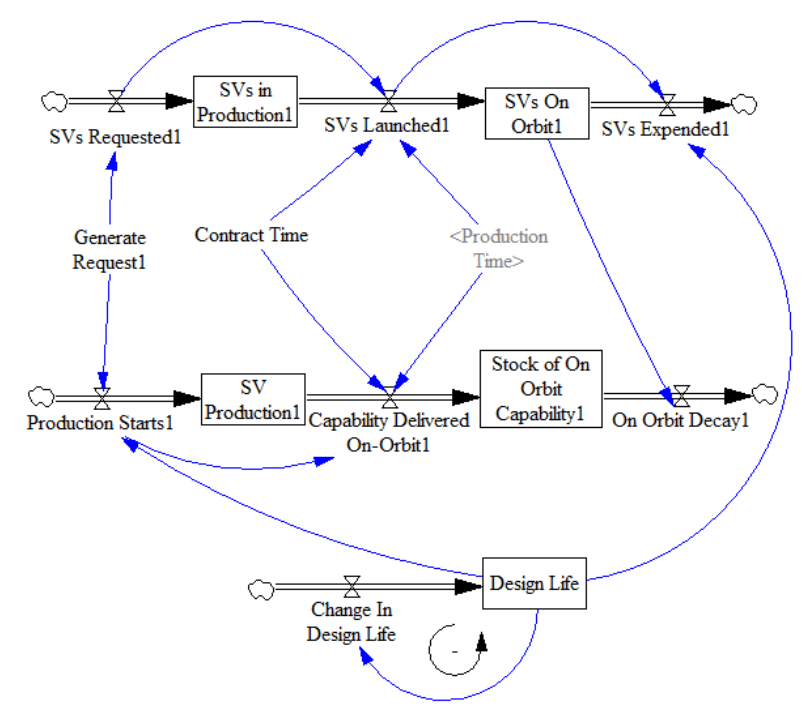




\section{Figure 4 Satellite Production Line Model}

Next, the structure represented in Figure 5 was adapted to this problem from Morrison's [30] work on learning in manufacturing. This is one way to represent the learning and forgetting within a process; a model of learning effects and their strength over time based on learning curve theory. This structure states that activity creates new experience over time, while experience degrades as time moves forward. Based on the level of the Experience stock the concept of a Learning Curve can be set where higher experience improves learning effects and entropy degrades learning effects. One might view this, as saying as technology progresses old technology is not as valuable, and as people change in and out of the industry individual skill is lost. The rate at which learning progresses or is lost is in relation to the "Half-Life" of the process. Learning occurs when satellites are produced. In this model both learning and forgetting for the industry as a whole as well as learning for the specific program, GPS, under evaluation must be tracked. The concept of learning with respect to satellite construction is represented in two forms in this effort; reductions in cost and schedule when creating clones of an existing system, and reductions in cost and schedule when creating new systems. As such this modeling effort must possess the ability to examine how these variables can change over time.

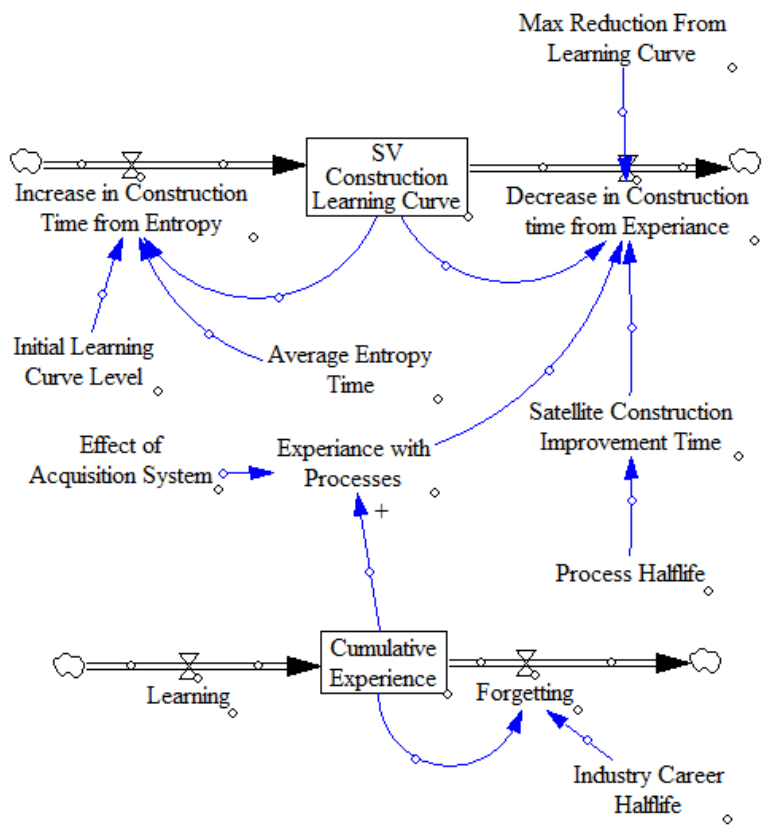


Figure 5 Experience and Learning Effects for Satellite Construction

It is important to be able to model the efficiency as work is being done. According to Morrison[30] the rate at which work is completed is defined as Resources multiplied by the efficiency with which the (limited or constrained) resources are implemented based on an "error rate" or "rework fraction." Theoretically, given a set process, people have a level of familiarity with that process, and the more familiar they become the more they can achieve with the same resources. The Rework Stock creates a "tug of war" between one and zero (i.e., the max and min error fractions), where a zero denotes that no work ever completes and a one is the perfect "happy path" where no mistakes are ever made in production. Both are impossible states in both reality and the model.

To adapt this concept of a pipeline production and the process of producing widgets to the process of building satellites, several modifications are needed. The first modification is for this model to allow the variables Cycle Time, Process Improvement Time, and Rate of Entropy to change over time. Cycle Time or Process Improvement Time was considered an exogenous constant in Morrison's work because his model was applied to workers adapting a specific process with a known development time. Due to the shorter time horizon of his work it was also assumed that the rate at which entropy was introduced into the system could be considered to occur at a fixed rate that would remain unchanged over the time period of the investigation. In Figure 6 both of these constants are modified and shown as variables that change throughout. This is necessary because the time period of investigation extends over a long enough time horizons that these two variables 
(Entropy and Process Cycle Time) are likely to change.

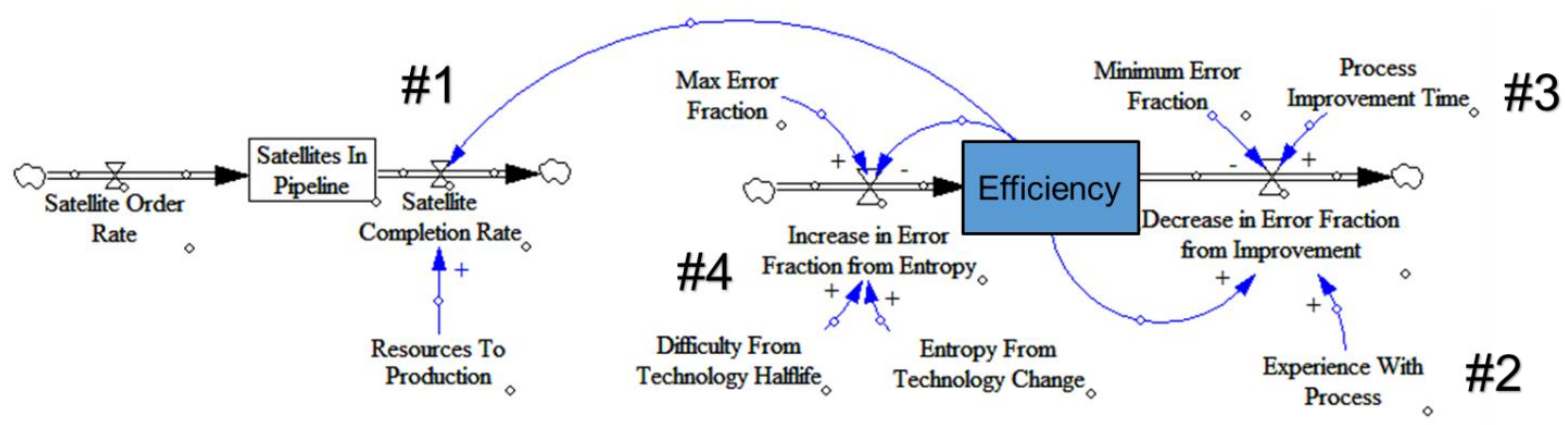

Figure 6 Efficiency Implementation

Rework is now dubbed "Efficiency," not just because Cycle Time and Entropy are brought into the model, but also because the work being completed is not whole satellites but rather, pieces of satellites. At any given time-step a whole satellite may not be produced, rather a fraction of one. (e.g., if the pipeline produces one satellite every six months then based on eight time steps per year each time step produces one-quarter of a satellite). In comparison, where Morrison's original work considers a single pipeline producing widgets, the GPS pipeline is now theoretically comprised of Work Breakdown Schedule (WBS) elements. The combined model will eventually use mass instead of widgets or WBS elements as it is a dynamic value that can be computed from existing models.

These concepts comprise their own causal loops, each passing through the Efficiency variable, hence making Efficiency the flow control variable that determines the rate at which resources (FY 2010 \$M USD) are converted into satellites. The model has two concepts, Entropy and Experience with Process. Entropy and the rate at which entropy advances makes work more inefficient. Experience and the rate at which lessons can be learned produce work more efficiently. These two concepts and their associated time constants (the time required to close half of the gap between the levels) form a balance over time in the Efficiency stock

Through use of these basic elements or molecules, the initial casual loops are turned into a working System Dynamics model with can be simulated and validated against real-world observed macro trends in this descriptive modeling effort. This System Dynamics model must now be tuned 
based on real world data such as number of launches or satellites per year, average design life, average mass, average performance etc. of satellites, in both the industry as a whole as well as the subcomponent under evaluation, which in this case is GPS. After this is complete the descriptive model can now be used to begin predictive modeling under specific context variables.

\section{Context Variables}

Context variables represent changes in the conditions in which a system operates specific to a time period and may be discrete levels or continuously changing functions. These variables are traditionally considered exogenous to Tradespace Exploration efforts. In this merged method these variables are represented by stocks in the System Dynamics model and are tuned based upon historical data. During model execution they will follow the trend line established by historical data but may also be changed based upon causal interaction among other variables. These variables must be considered with respect to the Space Industry as a whole as well as the satellite program being evaluated. Examples of such context variables in this effort are:

1. Exogenous technology: as captured it represents technology coming from Industry as a whole to the Space Industry.

2. Launch Costs: tuned to real world data assuming 2010 cost as a base line.

3. Ground Operation Costs: The SMAD[29] cost model states that each satellite on average costs $\$ 5 \mathrm{M}$ per year to operate.

a. This assumption must also be evaluated with respect to scaling of disaggregated assets. If each additional satellite adds the same cost to operations as the first, this cost could surpass acquisition costs.

4. Overall Activity Levels in the Space Industry: It is possible that the Space Industry could create more than, fewer than, or the same number of satellites as historically. This could also be modeled as the amount of money spent in the Space Sector. Historical data to tune to either condition is available, looking into the future this becomes a context variable. 
These context variables can be implanted as continuous or discrete functions either through look-up table or through a trend line. Trendlines, representing relative change over time can be more easily be understood by humans than a constant number as they speak to relative change over time.

Tuning Variables are artifacts of the modeling and abstraction process. These variables must be selected such that results of descriptive modeling efforts correspond to real world performance or are required to tie to abstract concepts together which have no physical meaning in the real world (e.g. experience). These variables can be sources of error and are traditionally the first assumptions to be examined in validation of any modeling effort. In this model examples such as the impact of the acquisition system on learning, Decay or Half-life constants for technology are examples of such variables.

\subsection{Tradespace Exploration}

\section{Design Variables}

Design Variables are those that fall under the control of decision or policy makers. These lead to the construction of the design vector which is the enumeration of all combinations of design variables; this is typically fed into a Tradespace Exploration for evaluation. One possible enumeration of the design variables produces what is referred to as a design points; the design vector contains all the design points[31]. The design vector in this paper contains only three variables and they have been tied to the GPS program selected for illustrative purposes. These variables have immediate impact on the cost and performance of a satellite and the constellation in which it operates but some may also have impact on subsequent generations.

As initial modeling seeks to compare the closest like-to-like comparison for performance and studies of disaggregation, orbits are not changed for the purpose of this study, as this would have 
cascading design changes that would inherently change the performance of the systems. Thus the orbits of all the satellites in this paper are fixed at the current GPS orbits in MEO[3]. This study uses the published design parameters of the GPS III program namely that it is to be built to last 15 plus years, contains seven payloads and will likely be purchased in three to four blocks of eight satellites[32].

The variables chosen for analysis in the design vector are Disaggregation, Technology Refresh, and Design Life. For the first variable of disaggregation, four permutations were instantiated. The model will either: keep all payloads on one bus (Full Aggregation), split into a military satellite and a civilian satellite (2X Disaggregation), split into a military satellite, an "old signal" civilian satellite, and a "new signal" civilian satellite (3X Disaggregation or the Galileo implementation), or Split every payload into a single satellites (Full Disaggregation). The second design variable of technology refresh is implemented to represent production of a new design. A new design would create a technology refresh event and incorporates the latest external technology into the design (e.g. processing, batteries, solar cells) and requires a new NRE. The optimal balance of how often to create new designs vs. replication of an existing design cannot be known before simulation. Short time intervals will cost more, as more NRE must be paid more often, and more designs will exist simultaneously. However, working with newer technology will have less legacy cost, higher performance and create more experience in design of satellites reducing NRE costs across time. Thus, the two choices are: clone the last block of satellites or pay NRE costs at the beginning of each block to upgrade to the latest technology. The last variable of design life impacts the trade space in several ways. Shorter life satellites are smaller weighing and costing less while forcing higher production quantities. In theory this leads to more experience and opportunities for technology refresh; this variable will have interaction with the second variable, tech-refresh. The shorter lived a satellite the more rockets are required in the same time period, which will lead to higher initial costs. This variable in existing cost models always optimizes at a single point in time to the longest life satellite possible, as cost can be spread over more years. In looking at multiple generations we 
are interested to see if this is still the case. In the data shown here only 15 year design life (the current design point) and 7.5 year design life (half the current design point) are shown, but many more options were evaluated.

This execution will produce a full factorial expansion of all design vectors at each acquisition decision. For the purpose of tree pruning and to reduce execution time, rules may be implemented within the construction of the design vector at each node. The Tradespace Exploration module follows a sequence in costing and evaluating the performance of each design at the given point in time based on current context variables provided from the System Dynamics model and is depicted in Figure 7. 


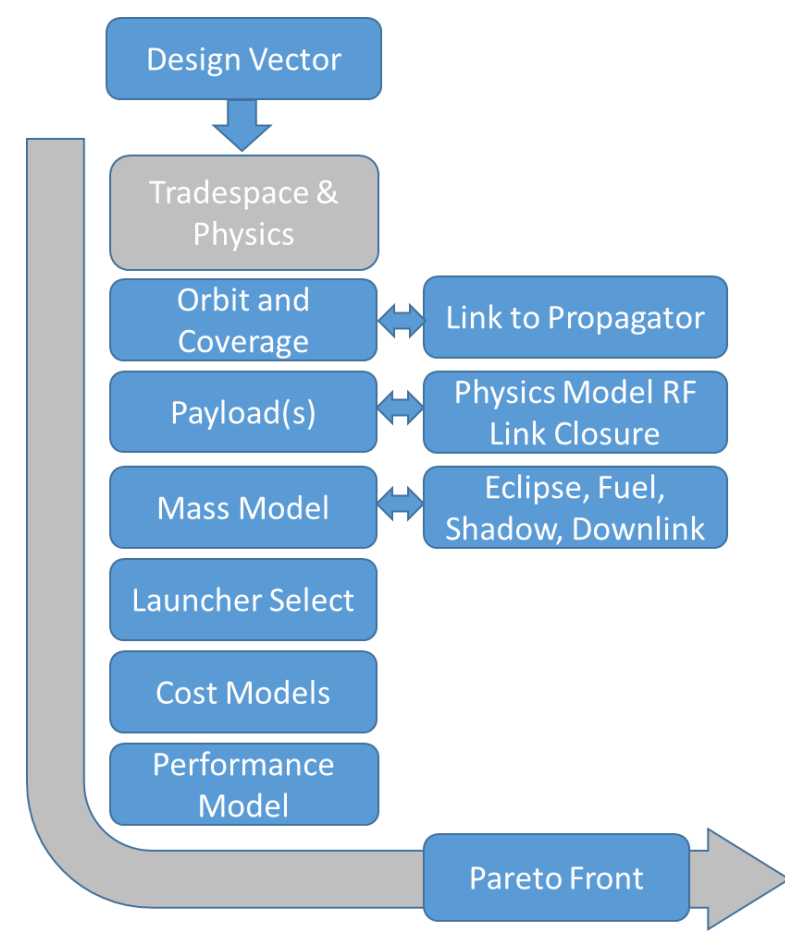

Figure 7 Execution Sequence of Tradespace Model

\section{Physics and Cost Models}

The most important physics model for the GPS example is the link closure algorithm: a GPS satellite is effectively a communication satellite that must close a link with a ground receiver (e.g., a cell phone, or other navigation enabled device). This requirement, given assumptions about data rate, path loss, antenna design, and other design parameters ultimately results in a power requirement for the transmitter which sizes the power system and has great impact on the overall size of a satellite. For the GPS example, the link budget is calculated from the equations listed in the SMAD[29] a method that has been validated by comparing Iridium's theoretical performance versus 
real world performance[33]. Tuning parameters and variables were derived from the GPS standard listed on the gps.gov website[3]. Information comparing relative sizes between the Position navigation and timing satellites mass and power requirements for GPS and Galileo are also available online[28]. Other Physics models that determine space or size, weight, and power are added as needed to enhance fidelity and trust in the predictive model.

Cost Models and cost estimating techniques for satellites can be found in the Space Mission Analysis and Design Handbook (SMAD). This provide an existing cost model to use in predictive modeling efforts for space systems

\subsection{Linking Tradespace Exploration With System Dynamics Variables}

The System Dynamics (SD) model stores the context variables for the Tradespace Exploration (TSE) model. In turn the decisions made in the Tradespace Exploration model change the context stocks over time. Thus, the variables that pass information must be determined. These include, for example:

- Production Time; depends on design life requested in TSE as well as the state of Learning and Experience in SD.

- Satellites on Orbit; represents the amount of capability on orbit at any given time. Serves as part of a proxy metric for evaluating survivability in a utility function.

- Stock of Capability (in operational years) on orbit; used to trigger the procurement when capability runs out.

- Learning Curve Effects; used as the exponential value in either cost estimation for the first unit produced or the reduction in cost in replication to the $\mathrm{n}^{\text {th }}$ unit.

- Stock of Capability (In operational years) in production; a proxy metric for the health of the Industrial base, this type of stock that can be used in a Utility function. 
- The Current Year in Execution; responsible for synchronizing context variables with time.

\section{SD Loops that Pass Through TSE}

As the primary goal in building a System Dynamics model is examining causal effects, and the goal of this work is demonstrating a method to fuse causal loops with Tradespace Exploration, two of the primary loops flow through the Tradespace Exploration Module. Some examples of such loops are:

1. Satellite production puts satellites on orbit (SD), satellites on orbit decay (SD), which triggers satellite procurement (TSE), which passes to the Tradespace Exploration module, which then places the appropriate number of satellites to be procured into generate request for satellites (SD).

2. A change in Design Life changes the process half-life of learning effects which changes the rate of decrease in learning, which affects production time, and in turn changes the point at which new satellites are procured (i.e., the longer it takes to build a satellite, the sooner new satellites must start production). The Tradespace Exploration model will then either keep the Design Life the same or change it before completing this loop.

3. A loop, with a very long time delay also exists when the concept of the entire Space Industry is examined. It postulates that learning on the GPS program correlates by some factor with learning in the overall Space Industry, which leads to reductions in cost and production time, leading to a better performing industry, which in turn brings knowledge back to the GPS program. GPS design vectors are produced in TSE where the entire industry is modeled in SD.

\section{Execution Sequence}

The overall data flow and execution sequence is illustrated in Figure 8 


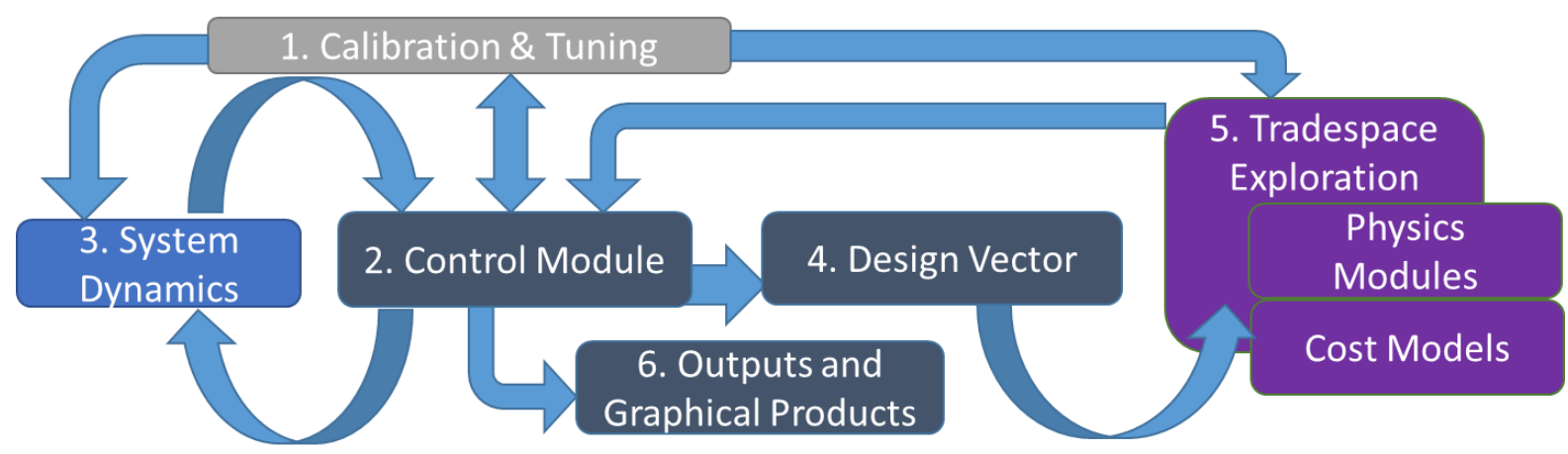

Figure 8 Model Data Flow

The model is written such that the Control Module handles execution flow, storage of data, and invocation of the System Dynamics and Tradespace Exploration models. Execution forms an everexpanding tree that, based upon the design vector defined might be visually represented as in Figure 9.

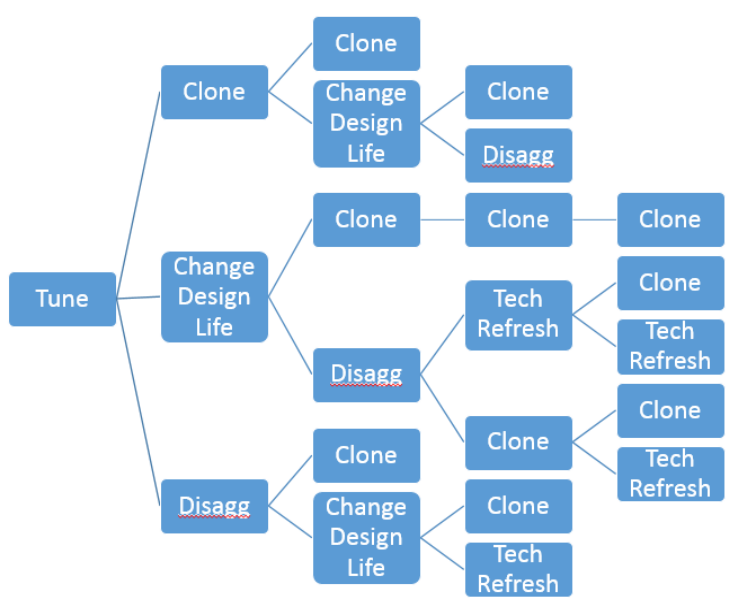

Figure 9 Possible Decision Tree of Future Acquisition Decisions

Calibration and Tuning runs the System Dynamics Model on historical data until the "time" where analysis is to begin; this trains the model based on tuning data. This "time" does not need to 
be present day to examine historical "what-if" questions, one can stop training the model at the time selected to begin the historical analysis. For the example below, the period of performance is set from 2000-2010 and to serve as historical data, the GPS block II satellite data is loaded during this time period. This model tuning now corresponds to Level 1 Node 1 . The model then proceeds to shift control from the System Dynamics module to the Tradespace Exploration module when satellite on orbit need to be replenished and a new acquisition is required. The result of procuring and launching the GPS satellites from the combined model is shown in Figure 10.

In order to validate the SD model, it is important assess the statistical validity in matching reference modes. This is accomplished by matching the model to tuning data and then using the matched model to see how well it reproduces data that has been held back and not used in the tuning. The goodness of fit was assessed using the Mean Average Percent Error (MAPE). The fit when using the MAPE is acceptable in not only the tuning period (1988-2008) but also for the hold back data (20082015) for all five reference modes:

1. MAPE for Unit Cost: Tuning $14 \%$, Hold-Back $10 \%$

2. MAPE for Design Life: Tuning $21 \%$, Hold-Back $12 \%$

3. MAPE for Total Number of Satellites Produced $13 \%$, Hold-Back $15 \%$

4. MAPE for Production Rate per Year (Out) 38\%, Hold-Back, $22 \%$

5. MAPE for Space Vehicles on Orbit: Tuning $20 \%$, Hold-Back $15 \%$

The Production Rate per Year (Out) has the worst fit, but this is unsurprising due to the large oscillations in the initial IIA constellation launch; the model more closely aligns with reality as time moves on and is a much better fit in the hold back period. Most importantly the fit of these variables is better than the error associated with the USCM8 cost model; the cost model used in the cost analysis and often implemented in government cost modeling exercises. In USCM8 the lowest error of any cost model is $23 \%$ and the highest error is $39 \%$ (as can be seen in Table $11-9$ of the SMAD[29]). Thus, this SD model when used for predictive modeling performs no worse than and often performs better than existing cost modeling practices when approximating satellite cost based on weight. 
To achieve this fit, the model implemented the artificial Efficiency variable as a flow control on the GPS satellite pipeline. The Efficiency variable by itself cannot be compared for "goodness" from one program to the next as a direct proxy for health. In conjunction with the model, however, it does enable direct comparison of the replication cost of satellites at a fixed performance level.

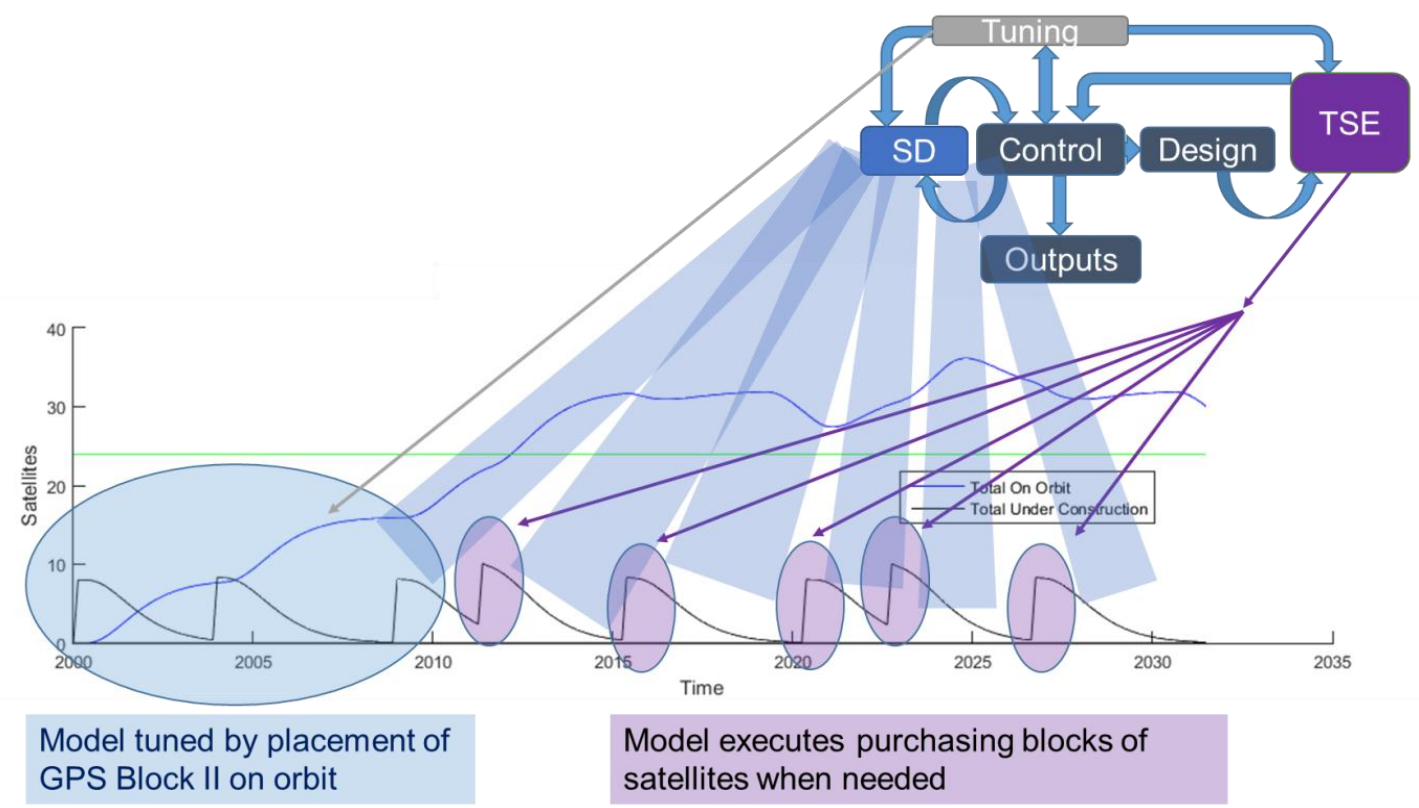

Figure 10 Model Representation of Procuring and Launching GPS Satellites under the current plan

\section{Results from variations on the GPS Acquisition Pipeline}

This work has created and validated a SD model that examines the procurement of GPS satellites over time, and merged it with cost and performance models within the framework of a TSE model. This merged model is now implemented to examine the consequences of various levels of disaggregation.

\subsection{Comparing Programs and Architectures Across Time}

A comparison of the cost effectiveness of programs and architectures across time requires a measure on which a comparison can be made. It was noted that the variable of cost per operational year is a potential metric. When this metric is extended to comparing programs across multiple 
generations, however, it can fail to provide an accurate comparison. Thus, cost per operational year is not sufficient as a measure for comparing cost effectiveness of programs.

Additionally, in comparing acquisition strategies, cost per operational year implies not just the cost per operational year on orbit but also the cost per operational year in a Five Year Defense Expenditure Plan (FYDEP). These are not the same variable. Cost per operational year on orbit refers to the number of years of capability derived from the total investment during acquisitions (and the on-orbit support cost), divided by the number of years on orbit for which the asset delivers value. Cost per operational year in the FYDEP refers to the amount of money spent in the current year to procure future capability. Spending in a present year in the FYDEP is important as it is the budget constraint in a current year, but the cost per operational year on orbit is not realized until the future (and really cannot be calculated until the satellite is taken out of service). The time lag between cost savings in the current year versus cost savings when satellites are on orbit can be a difference of decades. Thus, when comparing two acquisition strategies both cost metrics must be considered. It is possible that the implementation that minimizes the current year cost in the FYDEP may incur substantially larger costs in future years. It is also possible that the policy which decreases current year costs may produce less capability; and potentially in a non-linear fashion (e.g., saving $10 \%$ in the current year may cut more than $10 \%$ of the capability in years of operational capability delivered to orbit, resulting in an increase of more than $10 \%$ in the cost per operational year once on orbit).

There is a preference for keeping satellite production open and buying at a steady, predictable rate. One positive consequence is that this enables the SV builder to size a workforce and facilities for predictable acquisition levels. Additionally, and arguably more important, this style of contracting avoids the need to restart a closed pipeline in the event that additional unexpected capability must be procured, a process which can be extremely costly and time consuming. In practice an acquisitions pipeline is rarely closed until a follow-on contract is opened. In GPS, this is clearly seen where the production and launch time of the last IIFs coincides with the first IIIAs. It is also seen in 
the crossover of all 4 of the blocks in the II series (A, R, R-M and F). In the IIA to IIR transition, two of the IIRs were launched before the last IIA. There was a seven-month gap between the last IIR and the first IIR-M, and nine months elapsed between the last IIR-M and the first IIF.

Figure 11 has been constructed to illustrate these two problems. The model's forecast for the GPS BAU is displayed in blue, and a radically different and substantially more expensive architecture is shown in purple. The BAU is the Business as Usual case and represents the GPS architecture in the absence of any changes due to disaggregation, that is GPS II and GPS III[3] as currently planned and the best aggregated projections on them in future years. The colored squares represent the time for each acquisition, generations one through four. It can be seen that approximately four generations of the alternative acquisition occur within the same length of time as three generations of the GPS BAU. (This graph also illustrates the changing cost of production across generations). The problem of direct comparison is: should the first purple square be compared against the first blue square in terms of their production cost per year, or cost per operational year based on the capability put on orbit during their respective time periods? In point, the problem is even more complicated than just comparing across generation or time; it also extends to how many years of operational capability are acquired while spending resources.

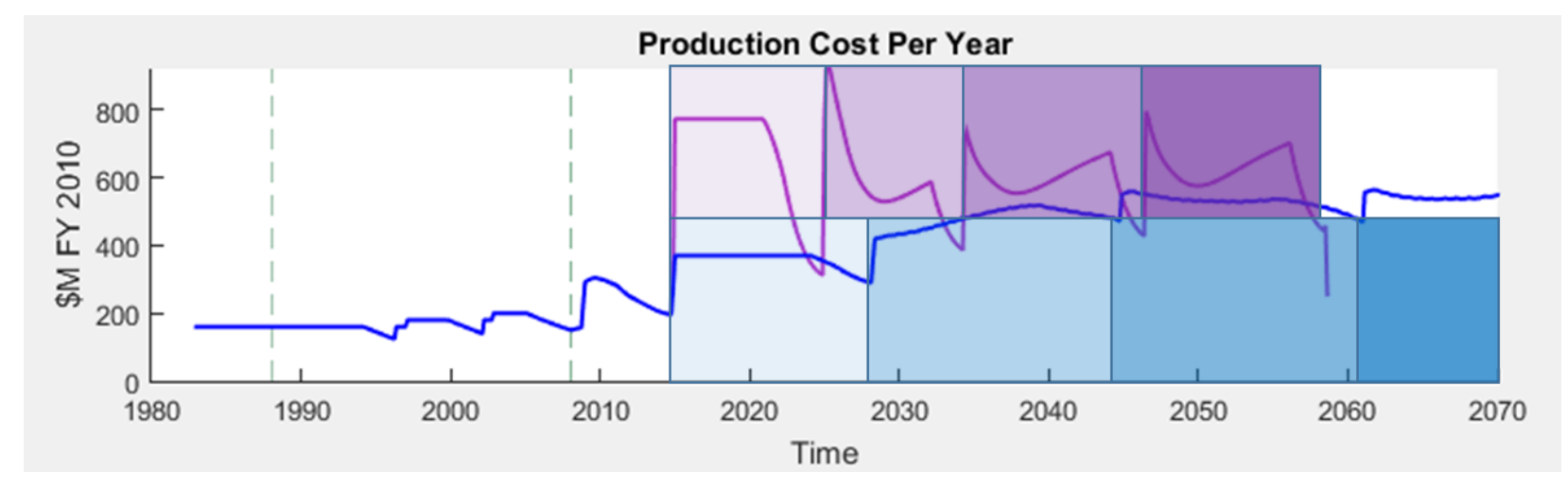

Figure 11 Comparing Acquisitions across Time for the GPS BAU vs. a higher cost and Faster

\section{Acquisition}


To enable appropriate comparisons of the different acquisition strategies considered in this chapter, results are presented for four variables that answer different questions about cost while providing the most direct comparison across time possible:

1. Cost per Production Year: This metric directly compares the total cost of the acquisition in a generation versus generation comparison. The total cost of all program elements are summed and divided by the number of years over which the program elements are acquired. The advantage of this approach is its direct comparison of the cost of two (or more) acquisitions. It does not consider time.

2. Cost to Acquire a Year of Operational Capability: The model assumes that every year the GPS pipeline must produce on average 24 years of operational capability in order to maintain 24 satellites on orbit. (Each satellite consumes one year of capability per year. The model targets 27 as the goal in production.) This metric compares how much is spent to acquire one year of on-orbit capability. A program with higher efficiency will likely perform better with respect to this metric. Nonetheless, while one program may be able to acquire capability at a more efficient level, since this metric does not consider time, it may also cost more on a per year basis (as noted for the first metric, Cost per Production Year). The appeal of this metric is that it speaks to the efficiency of the acquisitions system, lowest cost being best. This may or may not translate into overall savings, as one may spend more to capture greater efficiency. The decision-maker must decide if the premium over the GPS $B A U$ is worth the efficiency gained.

In this work, cost per production year, cost to acquire a year of operational capability, cost at the end of production and cost at the end of production adjusted for the difference of production rate will be used and compared to same numbers for the GPS BAU.

\section{Discussion of Assumptions Encoded in the Model}

The most significant assumptions are: 
- Disaggregation does not have part commonality among buses in the basic model. This biases the model to possess an increased cost in NRE for disaggregated implementations, as the cost for the buses themselves is computed as if each bus shared no components. This creates a conservative bias in the results.

- There is no complexity gain/penalty for integration and test based on the number of payloads. In theory one of the larger benefits of disaggregation is the shift (or even reduction) of complexity on the individual satellite. This complexity may shift elsewhere, such as the ground station, but it would be logical to assume that a satellite bus with one payload is easier to field than one with seven. While it may be countered that fielding seven satellites will likely be more expensive due to the quantity of activity; the model includes the increased difficulties in additional mass and cost for disaggregated policies while withholding a benefit from reduced complexity.

- The efficiency associated with the cloning and creation of satellites has been combined

- Performance of GPS has always been designed to be the best current technology can deliver

- The difficulty at any point in time to produce the best satellite remains static (1983-2045)

- Changes in technology and tools compensate for higher performance demands

\subsection{Single Variable Changes (Design Life, Order Quantity, Tick-Tock)}

\section{Change Design Life}

In this section the model was run with only the design life of the satellites changed. This is not a real policy that is being proposed, but rather is used for illustrative purposes to examine change across time. The blue line (as always) represents the tuned implementation of the GPS BAU. The red 
line represents a design life of 6 years, magenta 8 years, yellow 10 years, orange 12 years, and purple 20 years. In Figure 12 the production cost per year of this policy is change is seen.

The first observation about the figure is that, as expected, the satellites that provide the fewest years of capability also require the largest amount of money per year to produce equivalent years of operational capability. This is in keeping with standard satellite design and optimization as previously discussed with respect to current trends in the industry; the additional mass and complexity to extend design life is small relative to the years of on-orbit capability gained. The second observation from this figure is that the shorter the design life, the faster the acquisitions must come in succession and the faster each acquisition must complete. This is also logical, as from the variable Years of Operational Capability on Orbit we note that a satellite with 7.5 years of operational capability would only last half as long as one that possessed 15 years of operational capability, implying that in the same time period two satellites would be required to provide capability over the same time.

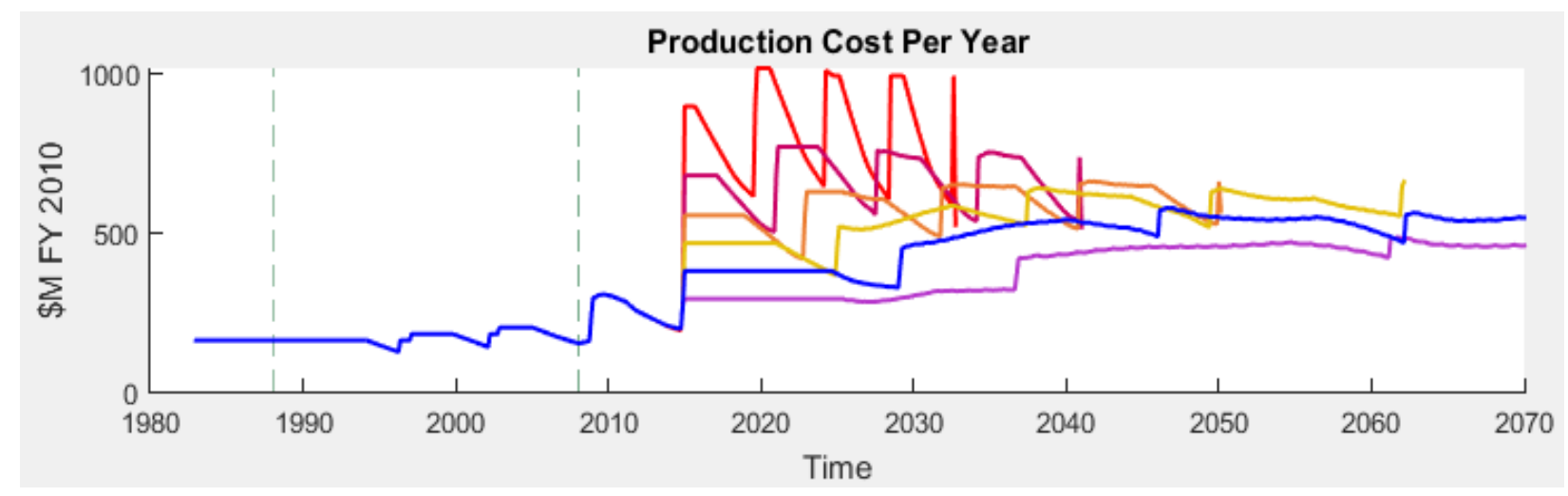

Figure 12 Changing Design Life $(6,8,10,12,15 \& 20$ years)

Table 2 presents a comparison of cost per production year for varying design lives. This table presents only the first cost metric, a generation-against-generation comparison. These results are an extreme comparison: the first generation for the design life of six years ends in 2019 and the first generation for the 20-year design ends in 2036. This concludes with a fourth and final generation for the six-year design life ending in 2032 versus 2079 for the BAU. While this comparison gives no 
deference to time, it is useful for examining the model's basic results, as this data should follow a predictable trend.

Table 2 Change in Cost per Operational Year with change in design life

\begin{tabular}{|l|l|l|l|l|}
\hline Design Life & Cost Change per & Cost Change per & Cost Change per & Cost Change per \\
& Operational Year & Operational Year & Operational Year & Operational Year \\
& Gen 1 & Gen 2 & Gen 3 & Gen 4 \\
\hline $\mathbf{6}$ & $189.48 \%$ & $144.70 \%$ & $136.49 \%$ & $131.21 \%$ \\
\hline $\mathbf{8}$ & $149.52 \%$ & $121.41 \%$ & $119.86 \%$ & $117.37 \%$ \\
\hline $\mathbf{1 0}$ & $126.12 \%$ & $107.70 \%$ & $109.50 \%$ & $108.59 \%$ \\
\hline $\mathbf{1 2}$ & & & & $109.06 \%$ \\
\hline $\mathbf{1 5}$ & $112.88 \%$ & $106.10 \%$ & $108.97 \%$ & $100.00 \%$ \\
\hline $\mathbf{2 0}$ & & & & \\
\hline
\end{tabular}

One would expect that two satellites of design life 7.5 years should (minus operational cost) cost $200 \%$ of one lasting 15 years. Interestingly, in the table the cost of the six- and eight-year design life satellites are both below $200 \%$ the cost of the 15 -year satellite design in the first generation. This reduction against the expectation is attributed to the efficiencies gained in production when greater quantities of satellites are built and there are faster refreshes of technology. Not surprisingly, either is a poor choice of policy as all the implementations cost more than the BAU (189\% for the six-year and $150 \%$ for the eight-year design life in the first generation). Across multiple generations, 
however, production cost does decline dramatically and the gap does narrow, though almost all the cost savings possible are gained by the second generation.

At the other end of the spectrum, one would expect that an increase of five years (from 15 to 20) would yield a $25 \%$ cost savings. Yet the table shows a cost savings of only $\sim 11 \%$ to $\sim 13 \%$. This is due in part to the increased mass of the longer lasting vehicles, but more importantly, it is a result of loss of production capability due to longer design life. It is for this reason that most of this paper focuses on policies that increase productivity. Eventually there will be a break point where design life cannot be extended to continue cost saving.

\section{Change Order Quantity}

We now turn to examining the impact of changing the quantity of satellites ordered from 24 (as with the GPS III BAU) to 12; half the current plan. This is also an interesting number because 12 is very close to the number of IIR, IIRM and IIF satellites actually produced, whereas the proposed current plan for Block III will reuse the bus for at least 24 satellites. This may give some insight into how procuring in different block sizes changes the cost of acquisitions. In Figure 13 the impact of the extra NRE events is clearly seen where the generation shifts occur more frequently. It can also be seen that the additional technology refresh dollars (one can think of this as acting as risk mitigation for long development items) speeds up the time between generations when compared to the BAU. Interestingly the overall cost of production is about the same across the same timeline with some small savings towards the end of each generation, but likely not enough to compensate for the $\$ 1 \mathrm{~B}+$ technology refresh cost. 


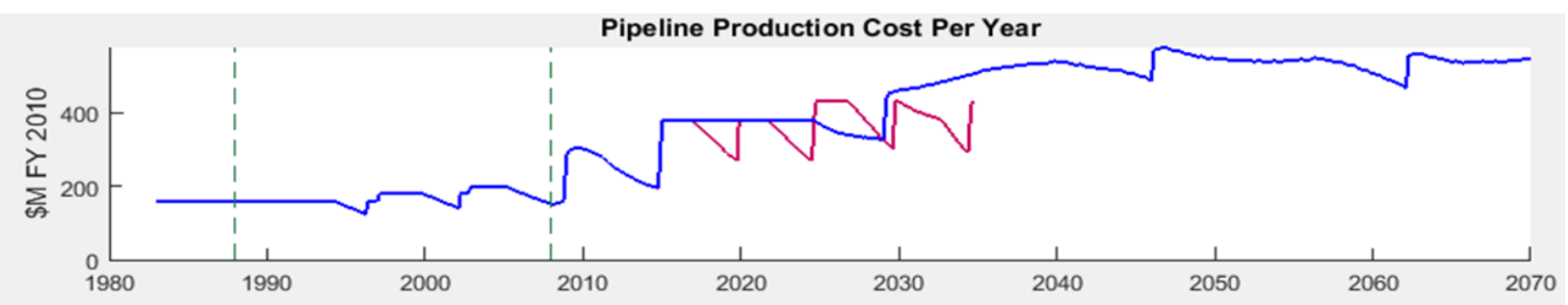

Figure 13 Changing Order Quantity (12 vs. 24 in GPS BAU) 
Figure 14 \& Figure 15 plot a dimensionless quantity representing a relative measure of the size of the satellites being launched across each generation against year. In these figures, if the line in one generation is twice the size of another then it can be concluded that the satellite of that generation have twice the wet-mass of the satellites in the first. Each spike in a colored generation is a satellite having been produced. Thus the figures display the number of satellites in a generation and the duration of the generation's production run. Figure 14 displays each satellite launch for four future generations of purchases in blocks of 12 . The black bars indicate the years in which the NRE pulses will be required to implement block buys of 12 satellites - they are 7.5 years apart. Comparing Figure 14 with the launch schedule for the GPS BAU (blocks of 24; NRE pulses represented by black bars 15 years apart), several differences are seen. The major difference is the completion date of the second generation for the order quantity of 12 (2025) versus order quantity of 24 (2028). Another major difference is the time between the NRE pulse of 2020 and first production of the Block IVs. With the GPS BAU, the model forecasts that it will take approximately nine years. The time is reduced to approximately six years with purchases in blocks of 12 . One might explain this in three ways. With the knowledge that only 12 of this design will be ordered and that a new design will be arriving in six years, it is less critical to get every possible upgrade. It might also be that the additional $\sim \$ 1.2 \mathrm{~B}$ in NRE funding was able to assist with risk reduction and lead time development, thus reducing the amount of time required for development of the new design. Finally, it might be that as the time between generations is now only half as long, less technology needs to be reengineered and as less of the design changes it takes only six rather than nine years to build the theoretical first unit. 


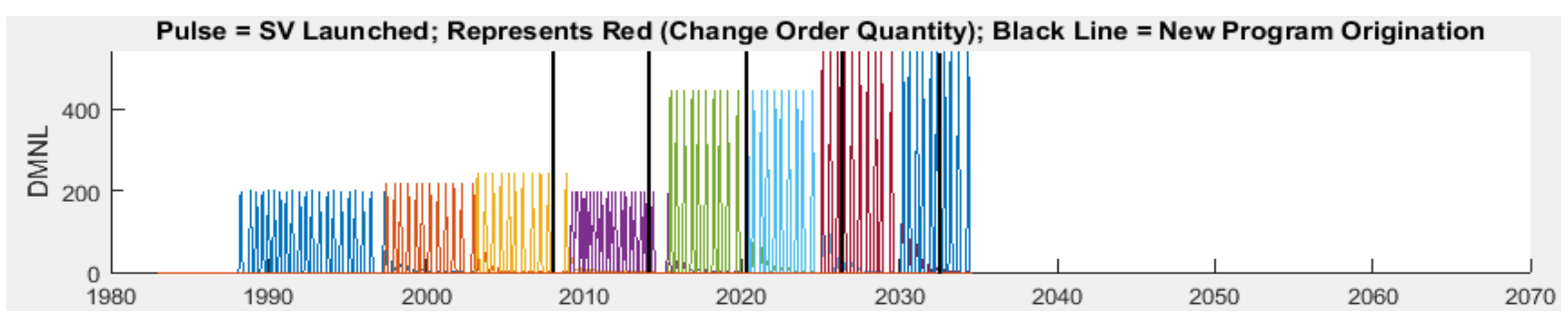

Figure 14 Satellite launches, order quantity 12

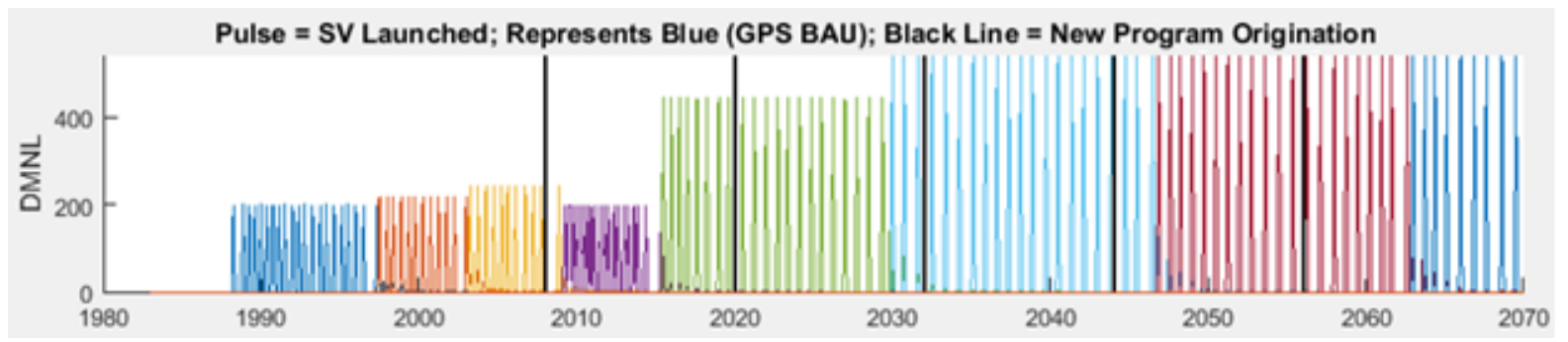

Figure 15 Satellite Launches, order quantity 24, GPA BAU

Note the first policy (block buy of 12) keeps the maximum 32 SVs on orbit, whereas the second (GPS BAU) cannot even maintain 24 without additional on orbit life. This is seen in the next figure.

However, the first costs more per year as it is not reaping the benefit of the emergent on-orbit life.

In fact while the production cost per year appears to be the same as the GPS BAU when considering the extra launch and production the cost of the quantity of 12 buys is between $155 \%$ and $161 \%$ that of the GPS BAU.

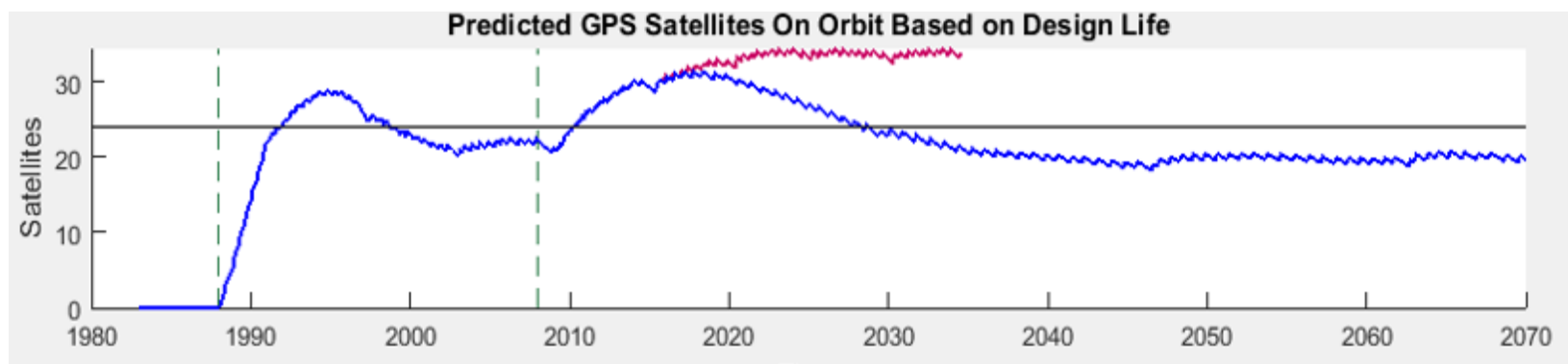

Figure 16 Satellites on orbit, order quantity and 24, the GPS BAU

\section{Tick-Tock Policy}

The Intel Corporation developed a policy of updating Architecture, then Photolithography offset by 18 months to create a three-year cycle for a complete micro-processor update. This policy known as 
Tick-Tock can be adapted to satellite design where interleaving the bus (tick) and the payload (tock), establishes a shorter time between NRE events to better combat entropy through technology advancement. In the next figure, the technology refresh pattern of the Tick-Tock program (red) is compared to the GPS BAU's technology refresh (blue). For the Tick-Tock model instead of a block of 24 satellites, two blocks of 12 satellites are implemented. This means that, all else being equal, two NRE pulses must occur for Tick-Tock in the same time period as one would happen for the GPS BAU. As the technology refreshed is only half of the technology in any single GPS BAU NRE event, the average technology never returns to "full" in the Tick-Tock implementation. The red line, representing the average decay of the technology of the GPS satellites in production notes the average number of half-lives that have elapsed and the amount (percent) of technology which must be refreshed at the next interval. Overall, the Tick-Tock policy when applied to satellite production smooths the average age of technology being used. This adds a constant level of difficulty in working with technology but avoids ever working with very old technology. This may be preferable to the expected BAU pattern of refreshing and working with technology that is three half-lives old.

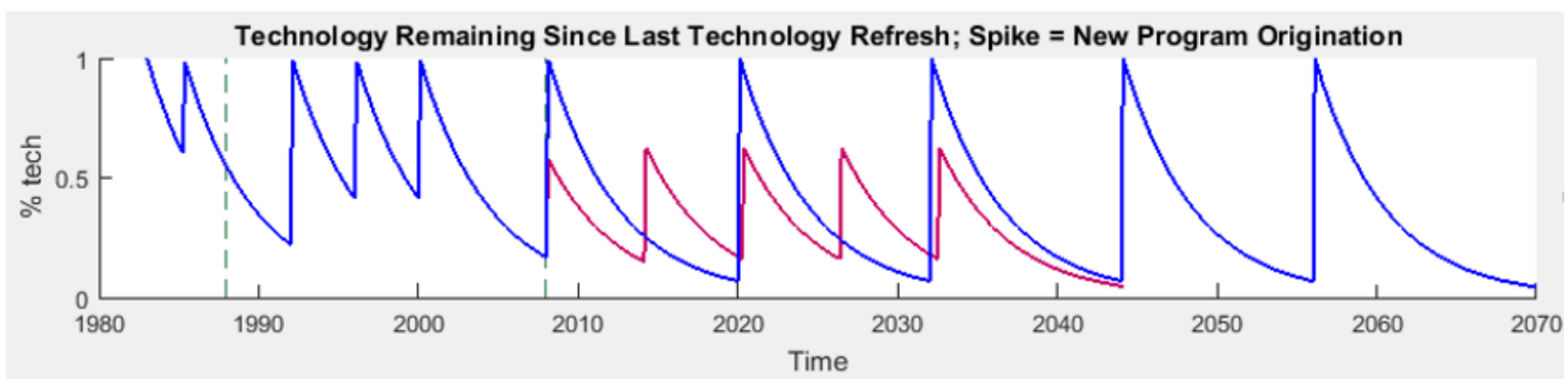

\section{Figure 17 Technology Refresh, Tick-Tock (12+12)}

The expectation of the Tick-Tock policy is to fix the Production Cycle Time in a uniform and predictable time sequence. In Figure 18 it can be seen that Tick-Tock does fix the Production Cycle Time into a uniform stable oscillatory pattern across generations as illustrated by the red line. Production Cycle Time for the GPS BAU is predicted to oscillate, but in an increasingly variable pattern. 


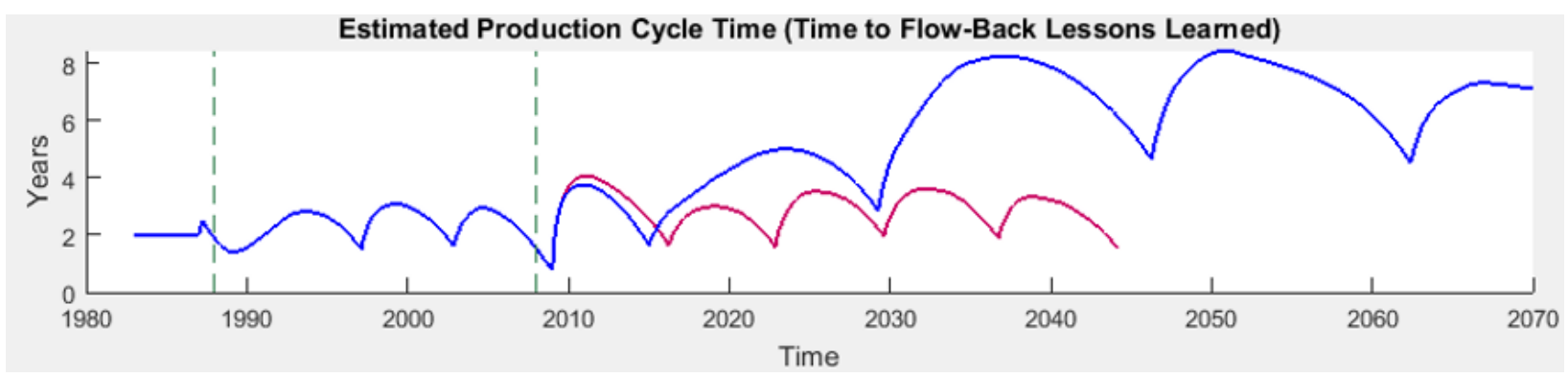

Figure 18 Production Cycle Time, Tick-Tock vs. GPS BAU

The next figure compares the Production Cost per Year for Tick-Tock versus the GPS BAU. Costs are less for Tick-Tock: the red line is under the blue line slightly in the first two generations and significantly lower in the second two. This implies that Tick-Tock can achieve cost savings over the BAU in production units despite requiring increased NRE in the first generation. The figure also shows that the first two tick-tocks take a year longer to complete than the GPS BAU but the second tick-tock (third and fourth generations) completes three years before the second generation of the GPS BAU. This indicates there will be some initial difficulty in implementing Tick-Tock, but speed in production within and across generations will improve over time.

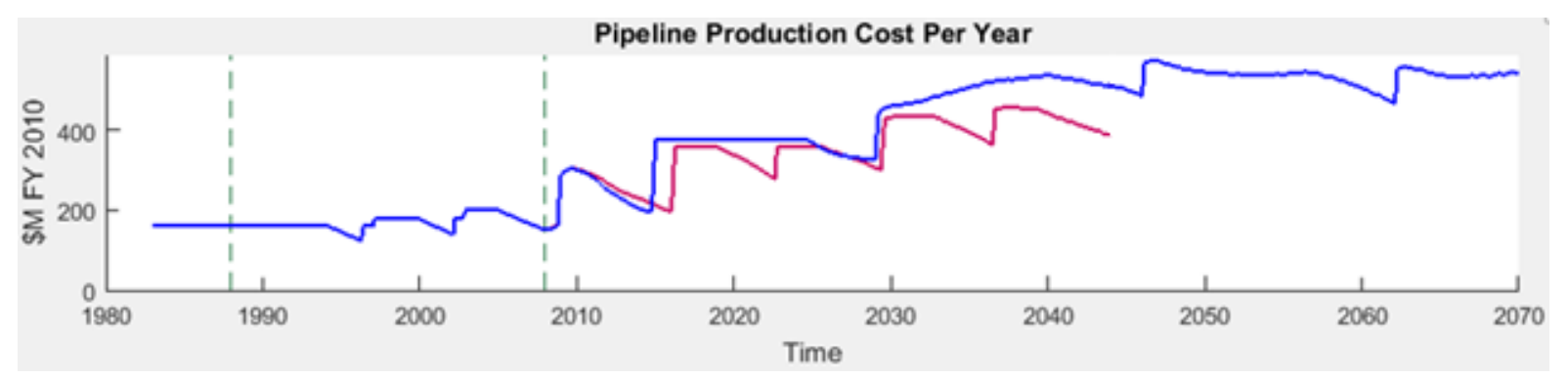

Figure 19 Production Cost per Year, Tick-Tock vs. GPS BAU

To compare the costs of Tick-Tock versus the GPS BAU, the four cost metrics are shown in Table 3. In the first generation Tick-Tock requires no increase over the FYDEP, and may achieve a greater efficiency-on the order of approximately seven percent-by 2030. We can expect that this greater efficiency will translate to more satellites on orbit for the same price. 


\begin{tabular}{|c|l|l|}
\hline S/N & \multicolumn{1}{|c|}{ VARIABLE } & \multicolumn{1}{|c|}{ Vs BAU } \\
\hline 1 & Generation Number(s), Design Life of Satellite (years) & Gen 1 \& 2, 15 \\
\hline 2 & Number Satellites Produced & $12+12$ vs 24 \\
\hline 3 & Average Cost Per Production Year Gen vs Gen & $99.41 \%$ \\
\hline 4 & Cost to Acquire a Year of Operational Capability & $93.38 \%$ \\
\hline 5 & Cost at End of Production, Policy Cost per Year Relative to BAU & $99.06 \%$ in 2030 \\
\hline 6 & Compensate for Difference of Production Rate (Time and Performance) & $92.98 \%$ in 2030 \\
\hline
\end{tabular}

Table 4 directly compares the third and fourth generation of Tick-Tock against the second generation of the GPS BAU. Improving over the first tick-tock, the second tick-tock (third and fourth generations) is forecast to cost four percent less than the GPS BAU while producing capability at a discount of $18 \%$ over the GPS BAU by 2044. In brief, the model forecasts for four percent less cost Tick-Tock could provide 18\% more years of operational capability versus the GPS BAU.

Table 4 Generation 3 \&4, Tick-Tock and GPS BAU

\begin{tabular}{|c|l|c|}
\hline S/N & \multicolumn{1}{|c|}{ VARIABLE } & \multicolumn{1}{|c|}{ Vs BAU } \\
\hline 1 & Generation Number(s), Design Life of Satellite (years) & Gen 3 \& 4, 15 \\
\hline 2 & Number Satellites Produced & $12+12$ vs 24 \\
\hline 3 & Average Cost Per Production Year Gen vs Gen & $96.14 \%$ \\
\hline 4 & Cost to Acquire a Year of Operational Capability & $82.10 \%$ \\
\hline 5 & Cost at End of Production, Policy Cost per Year Relative to BAU & $96.19 \%$ in 2044 \\
\hline 6 & Compensate for Difference of Production Rate (Time and Performance) & $82.04 \%$ in 2044 \\
\hline
\end{tabular}

These results indicate that if no other changes are made, consideration should be given towards implementing a Tick-Tock policy for the GPS acquisition pipeline. Yet, an examination of efficiency (see Figure 20) reveals an unfortunate reality. This policy has not "fixed" the underlying issues within 
the industry as modeled. The erosion/decay in Efficiency continues unabated. Tick-Tock treats the symptom but not the disease underlying lack of efficiency with resources in the industry.

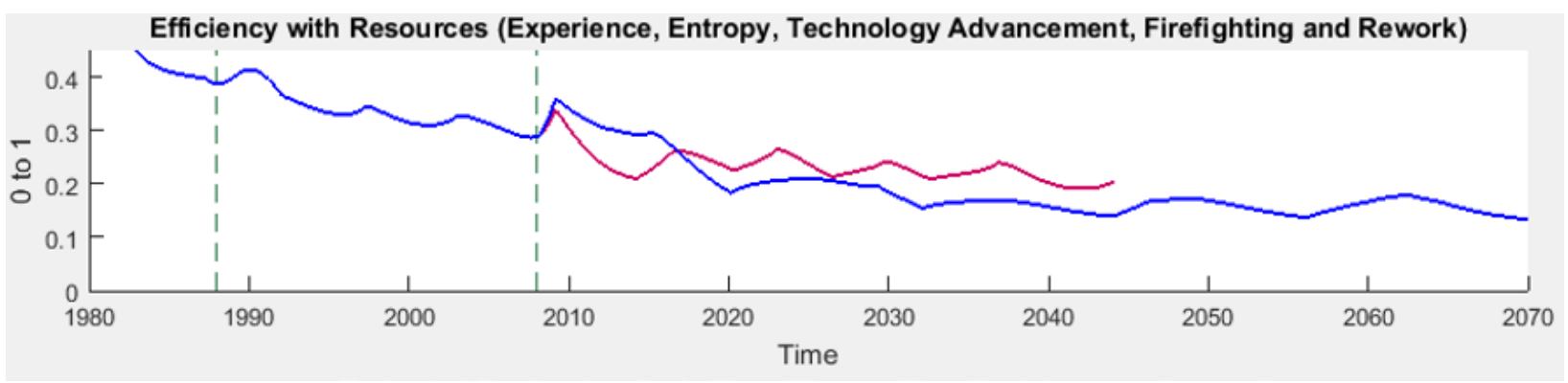

Figure 20 Efficiency, Tick-Tock and GPS BAU

\section{Disaggregation}

Previously in examining the consequences of a Tick-Tock policy, we noted that an initial investment of extra NRE created stability of the Production Cycle Time. Since this stability did not translate to a shortening of the time required from NRE to first production unit over the four generations, this indicates that Tick-Tock did not initiate a virtuous cycle, but rather was being kept in balance by the additional capital. Removal of the additional capital would return the acquisitions process to its unstable state. We will now examine Tick-Tock in combination with disaggregation, with an eye towards the same variables: shortening of time between NRE and first production unit, the stability and magnitude of Production Cycle Time and the total cost of production. We want to see if any disaggregated approaches can make the major SD loops work for the acquisition system and address the underlying issues which lead to decreasing efficiency both in and across generations of satellites. Can a Tick-Tock policy and disaggregated architecture achieve a short Production Cycle Time and higher Efficiency for no additional cost, or will higher Efficiency always exact a higher price within the current state of satellite procurement? In this work, four flavors of disaggregation have been examined. The plots in this section use the following color scheme:

- Blue: GPS BAU (as always)

- Yellow: Military/Civilian Implementation (2x Disaggregation) 
- Orange: Galileo-Size Implementation (3x Disaggregation)

- Red: Full Disaggregation (5x-6x-7x)

- Purple: Survivable Disaggregated (4x-5x-6x)

These arrangements were computed to deliver equivalent satellite performance with respect to the number of signals and signal strength delivered to Earth. The upgrade paths across time vary for each disaggregation approach. Upgrade paths were designed such that each generation would obtain new capability near in time to when the GPS BAU is forecast to obtain new capabilities, however, due to the causal nature of this work and the uneven program start and stop times it was not always possible. These arrangements are recapped here:

- GPS BAU: More signals are added to the single bus as performance increases are desired.

- Military/Civilian Implementation (2x Disaggregation): If the signal is for military purposes it is placed on the military bus, otherwise it is placed on the civilian bus.

- Galileo-Size Implementation (3x Disaggregation): One bus becomes the "Legacy Signals," the second becomes the "New Civilian Signals" and the final bus becomes the "Military Signals and Capability."

- Full Disaggregation (5x-6x-7x): Add another satellite with a single signal to the constellation. The reason for $5 x-6 x-7 x$ is each generation must add an additional 24 satellites for every signal added.

- Survivable Disaggregated $(4 x-5 x-6 x)$ : Another satellite is added with two signals on the new buss. The upgrade path continues the trend where the dual status of each signal is always maintained and two satellites always have each signal.

- This implies that at any time there are 48 transmitters for each signal on orbit, double the minimum threshold capability.

- The arrangement of payloads to buses enables no loss of capability in the loss of up to $25 \%$ of on-orbit assets. 
- Beyond 25\% losses satellites can be moved within their planes to close gaps in coverage up to $50 \%$ loss of on-orbit assets. This depends on which satellites are lost and in which planes.

- Depending on the satellites lost beyond $50 \%$ losses some capability may be maintained.

In addition to cost savings or efficiencies in production, disaggregated approaches offer other potential advantages, foremost being increased resilience. In a disaggregated approach it is assumed that similar to the GPS BAU, 27 satellites are the desired equivalent capability to keep on orbit. For either approach, up to three satellites might be removed and full coverage be maintained (given that 24 is the threshold for performance). In these disaggregated implementations it is reasoned that the loss of any individual satellite (below the threshold of 24) will create a capability gap only for the signals on the satellite that is lost. It is left to future work to determine the specific probabilities of loss of capability associated with removing various satellites on orbit.

In Table 5 the number of satellites required in each generation for the BAU and each disaggregated approach and the minimum number of satellites to maintain equivalent satellite capability is listed.

Table 5: Number of Satellites Required on Orbit per Generation in Disaggregation for Capability Equivalent to GPS BAU

\begin{tabular}{|l|l|l|l|l|}
\hline $\begin{array}{l}\text { Number of Satellites } \\
\text { Required on Orbit per } \\
\text { Generation }\end{array}$ & Generation 1 & Generation 2 & Generation 3 & Generation 4 \\
\hline BAU & 24 & 24 & 24 & 24 \\
\hline $\begin{array}{l}\text { Military/Civilian } \\
\text { Implementation } \\
(2 \times \text { Disaggregation) }\end{array}$ & $24 \times 2=48$ & $24 \times 2=48$ & $24 \times 2=48$ & $24 \times 2=48$ \\
\hline $\begin{array}{l}\text { Galileo-Size } \\
\text { Implementation } \\
\text { (3x Disaggregation) }\end{array}$ & $24 \times 3=72$ & $27 \times 3=72$ & $24 \times 3=72$ & $27 \times 3=72$ \\
\hline
\end{tabular}




\begin{tabular}{|l|l|l|l|l|}
\hline $\begin{array}{l}\text { Full Disaggregation }(5 x- \\
6 x-7 x)\end{array}$ & $24 \times 5=120$ & $24 \times 6=144$ & $24 \times 7=168$ & $24 \times 7=168$ \\
\hline $\begin{array}{l}\text { Survivable Disaggregated } \\
\text { Implementation }\end{array}$ & $24 \times 4=96$ & $24 \times 5=120$ & $24 \times 6=144$ & $24 \times 6=144$ \\
\hline
\end{tabular}

Figure 21 displays the production rate per year for the various flavors of disaggregation. Each of the generations orders satellites at the constant rate needed to keep the equivalent of 27 satellites on orbit at all times (with a minimum acceptable level of 24). The most obvious feature is the early spike associated with Full Disaggregation (red line). 3x Disaggregation (orange line) and $2 x$ Disaggregation (yellow line) follow the expected pattern in terms of quantity where orange is second highest and yellow is below orange. Upon closer inspection, it can be seen that each of the flavors of disaggregation tends to bunch up. The reason for this is fairly straightforward; the first few satellites take longer to produce than later ones. As the last satellites in a generation are produced, there is a hold-over period before production can fully ramp up for the next generation. Due to the oscillatory nature of this bunching it is interesting to see that sometimes the yellow line crosses over the orange line. One would expect that in a stable pipeline this would never happen, however, as generational changes act as large exogenous shocks the model encodes this variance. Not surprisingly, Full Disaggregation is associated with greater variability with respect to production rate out per year than any other flavor of disaggregation. All flavors of disaggregation achieve greater production than the BAU. The model predicts that at no time does production drop to zero. Due to the fact that the shock of switching to a disaggregated approach is greatest in the first generation, the rate of output is most uneven in the first generation. As the model reaches stability by the fourth generation, the production rate out stabilizes. (The purple line representing survivable disaggregated architecture has been removed from these initial graphs and will be discussed separately below) 


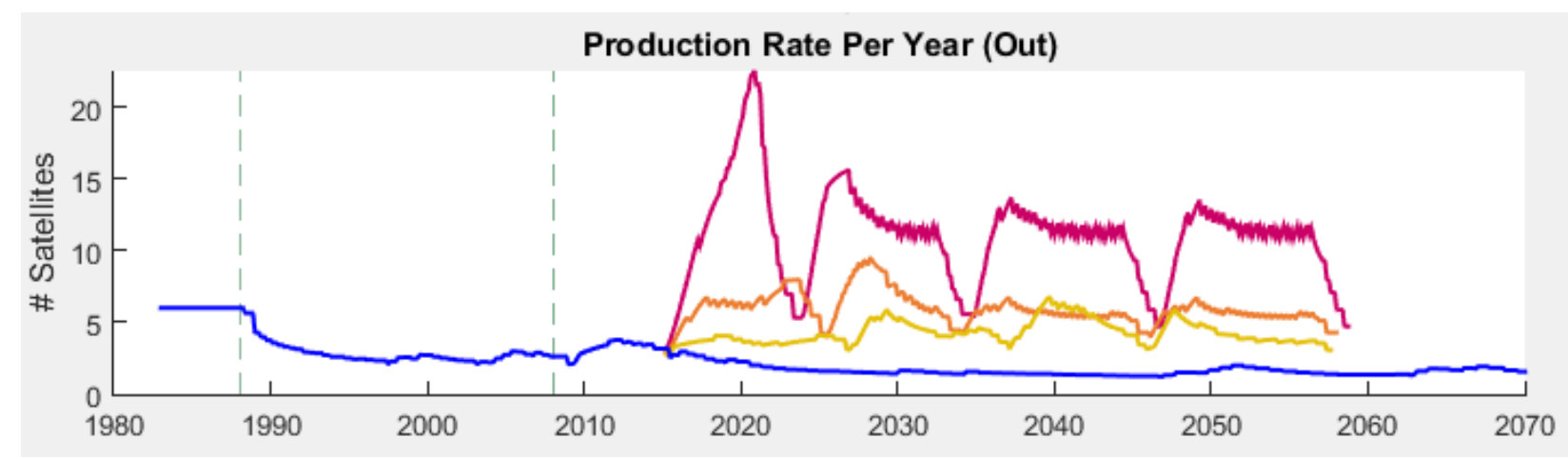

Figure 21 Disaggregation, Production Rate per Year

Figure 22 displays the equivalent number of satellites that must be kept on orbit for the various flavors of disaggregation. Note that with the disaggregated approaches, capacity is initially overshot, but eventually stabilizes. The most important finding is that for all of these approaches the number of satellites kept on orbit is always above the critical threshold (shown by the horizontal lines), implying that all three approaches would eliminate the need for satellites to last beyond design life in order to meet the performance threshold.

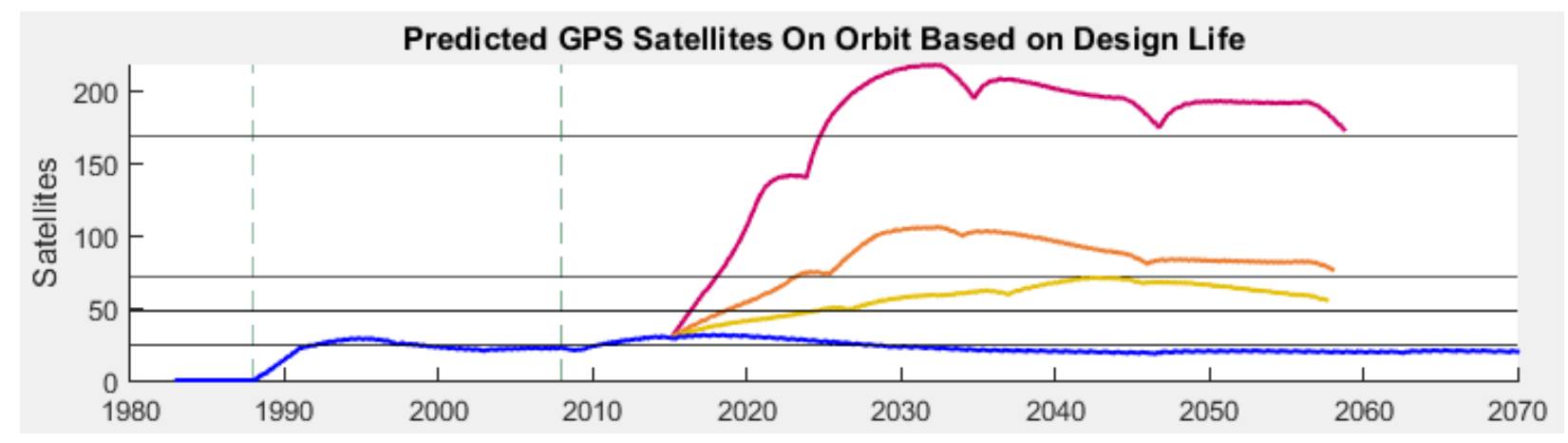

Figure 22 Disaggregation, Number of Satellites on Orbit

Figure 23 tracks the Production Cycle Time for satellites across multiple generations. The Full Disaggregation approach drops the cycle time to approximately six months inside the first generation, that is, over a span of 10 years. This is likely because Full Disaggregation involves relatively small satellites ordered in relatively large quantity. The Galileo-sized implementation is 
almost able, in a single generation, to drop the Production Cycle Time to its minimal level (seen in orange). In this model a policy has reached its maximum effectiveness when it has stabilized to the minimal level achievable. A policy that can transition more quickly to its stable region will be faster to provide cost savings in reproduction. In addition, a policy with a lower Production Cycle Time can theoretically capitalize on learning and transition lessons learned back to the next unit faster. While the graph depicts the disaggregated approaches achieving low Production Cycle Times (unlike previous policies examined), they are not saturated. The model is operating within a valid region and a different policy could in theory drive the Production Cycle Time even lower.

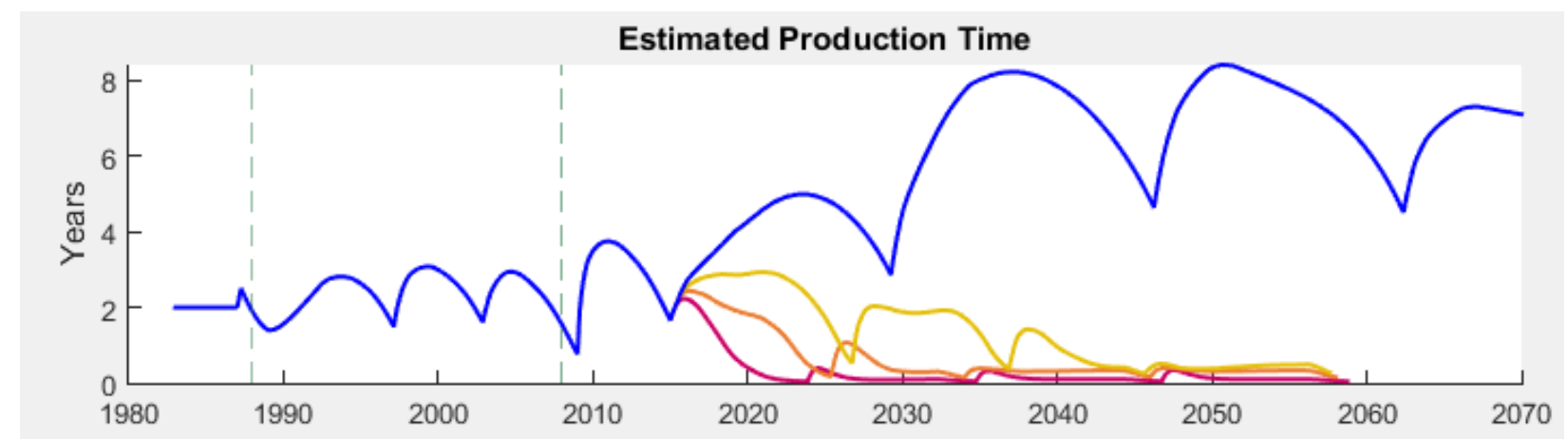

Figure 23 Production Cycle time for different flavors of disaggregation and GPS BAU

Figure 24 displays the relative production cost per year for each of the disaggregated implementations. One can visually extract the end time of each generation from the spikes in funding after the drop-offs in production costs toward the end of each generation. Examination of the figure shows four generations of disaggregation completing in the same time as three generations of the GPS BAU. This occurs because disaggregated approaches are able to complete acquisitions on time, or even early. As generations must sequentially follow in order to capitalize on learning effects across generations, the next program starts after the preceding finishes. This has the result of potentially faster generations. Most importantly, we see that by 2035 the production cost for each of the disaggregated architectures is below that of the GPS BAU. This indicates that by 2032 changes in experience and cycle time for disaggregation should be able to compensate for the additional quantity of production required. (This graph includes program overhead and 
integration/test activities, but does not yet factor in NRE, Launch, or Ops costs.) Also of interest is that while the $3 x$ disaggregated approach (orange line) is less expensive in the second generation than the $2 x$ disaggregated approach (yellow line), by the fourth generation this reverses and the $2 x$ costs slightly less than $3 \mathrm{x}$. It would appear that the model forecasts that the difference between 48 versus 72 satellites in a generation can cause a faster transition to the equilibrium state (maximum effect from policy change).

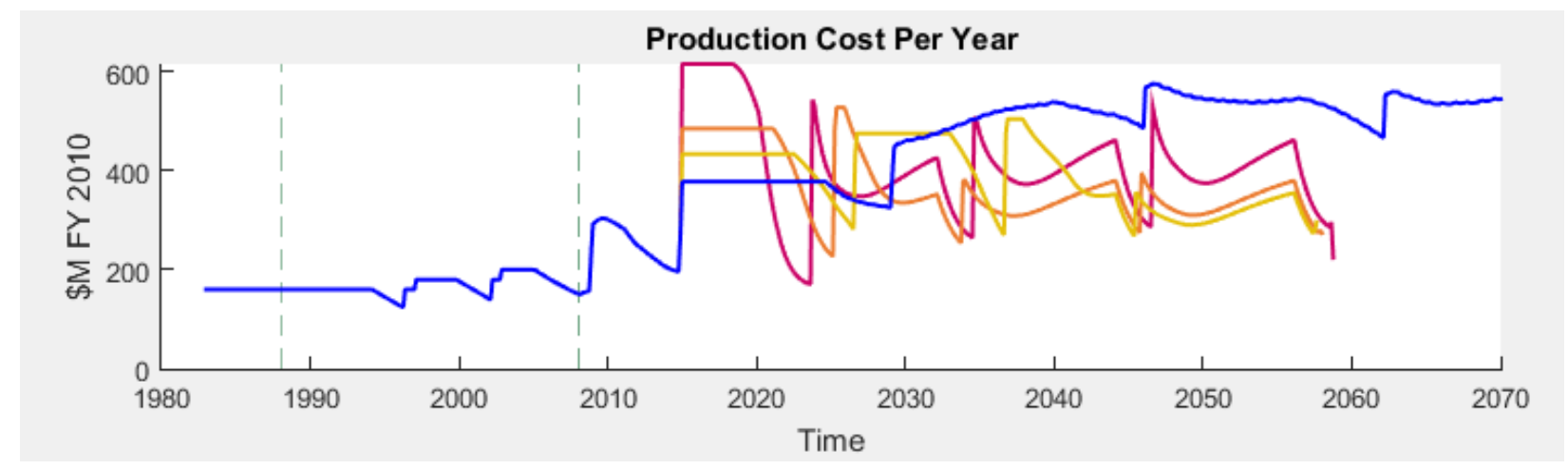

Figure 24 Production Cost per year for different flavors of disaggregation and GPS BAU

Figure 25 presents the relative efficiency of various approaches to disaggregation in production of GPS satellites. There is clearly a substantially non-linear gain from the difference between $3 x$ and $7 x$ Disaggregation. An interesting observation involves the $2 x$ Military/Civilian approach to disaggregation (yellow line) that slowly creeps up to its maximum efficiency over the course of all four generations. This slow creep explains the behavior of cost decreasing seen on the yellow line, generation across generation, seen in the production cost per year. The 7x Disaggregation involves so much activity that it can in one generation achieve its highest level of efficiency; the $3 x$ Disaggregation can reach its highest stable point in two generations and 2x Disaggregation achieves equilibrium in three generations. The trend of erosion in efficiency in the BAU (blue line) is reversed by all tested disaggregation strategies. This is clearly linked with the data of decreasing Production Cycle Times seen in Figure 23. One might ask, which comes first? The answer is neither; the additional experience in combination with the smaller size of more quickly manufactured satellites 
slowly pushes the model to get the reinforcing loops to work for, rather than against production.

Eventually the Production Cycle Time becomes short enough that learning is transferred quickly and the system becomes robust to the shock of transition across generations.

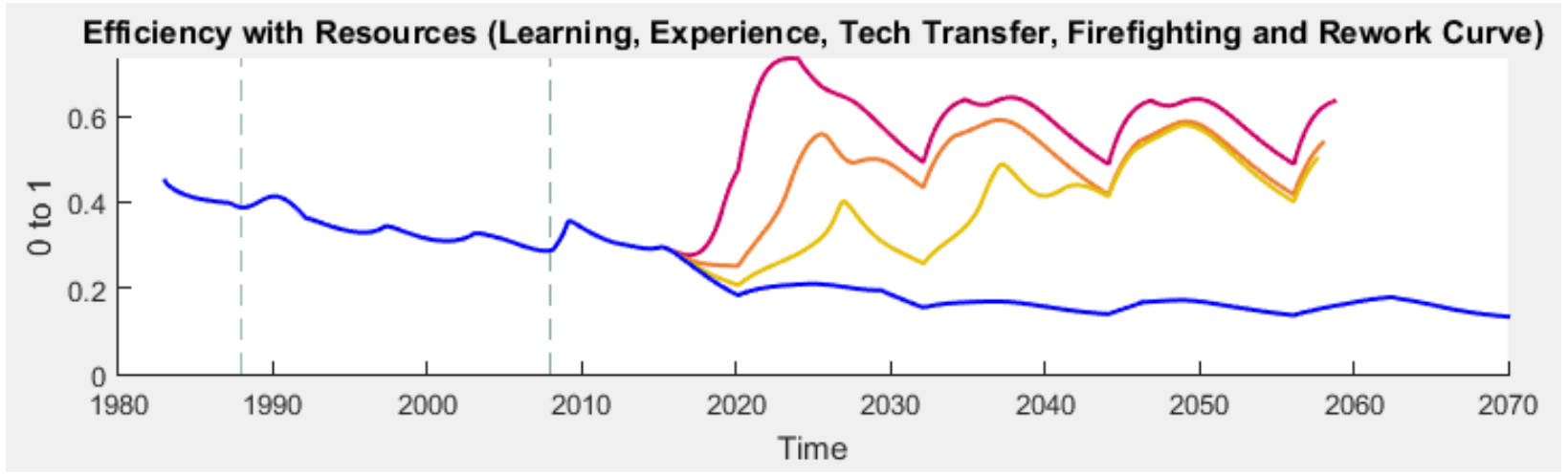

Figure 25 Efficiency with Resources for different flavors of disaggregation and GPS BAU

\section{GPS Disaggregation Results}

The GPS BAU is the baseline to which all disaggregation strategies are compared. In all initial analyses of disaggregation strategies versus the GPS BAU, the model is biased to favor the BAU. For example, no part commonality is assumed for the multiple satellites in these initial policy comparisons with the GPS BAU. Model bias in favor of the BAU implies performance will be no worse than computed for any policy of disaggregation and could be substantially better. After the initial analyses, effects of altering certain assumptions that favor the GPS BAU are summarized.

\section{2x Disaggregation}

Table 6 presents the results for splitting the GPS satellites into two (e.g., a military satellite and a civilian satellite). The following information is helpful in interpreting the data in this and following tables. 
- The first row (e.g., 2x Disaggregation versus GPS BAU): directly compares the tested disaggregated approach with the GPS BAU. The percentages in the upper sub-row should be interpreted as the average relative change to the FYDEP over the generations listed. The time period over which this comparison holds true is also listed to give the reader an understanding of the time over which the optimal policy operates.

- The percentages in the lower sub-row represent the cost to deliver equivalent capability on orbit. If this number is less than $100 \%$, then there is extra capability being delivered on orbit for the change in FYDEP cost above it. Often it is seen that the FYDEP requires an increase over the GPS BAU, but because the cost per operational year goes down, more years of operational capability on-orbit could be delivered than with the GPS BAU. For GPS this would imply more transponders on orbit. The additional years of capability delivered to orbit is, on-average, the inverse of the percentage in the lower sub-row. For example in Generation 2, roughly 29\% higher FYDEP cost delivers about 37\% more years of operational capability.

- In some cases, both numbers are below $100 \%$-these are very desirable outcomes in terms of performance and cost.

- Excluding operations costs: The FYDEP cost compared to the BAU but computed without consideration of on-orbit operations costs. The difference between these values and the first row above reveals how much of the difference between the tested policy and the BAU is attributable to operations costs associated with the change in the number of satellites being flown.

- Excluding launch costs: The FYDEP cost compared to the BAU but computed without consideration of launch costs. The difference between these values and the first row reveals how much of the difference between the policy change and the BAU is attributable to launch costs associated with the change in the number of satellites being launched and the change in the mass being placed on orbit. 
- Assuming part commonality and reasonable reduction in launch costs over time: Initially the model assumed static launch costs across all time and no part commonality among disaggregated designs. Here a part commonality of $50 \%$ is assumed and a tapering of launch costs is implemented. The tapering of launch costs is based on the following model. In 2020 launch costs come down $10 \%$ and by 2045 have reduced $40 \%$, with a final $50 \%$ reduction being achieved in 2090. It is possible that over time launch costs may become lower. For example, SpaceX was able to successfully place the SES-9 communications satellite with a wet-mass mass of $\sim 5000 \mathrm{~kg}$ into geosynchronous orbit on March $4^{\text {th }} 2016$ [34]. This implies that a SpaceX launch vehicle is capable of putting any of the disaggregated satellites envisioned in this work into a MEO orbit. Since SpaceX launches are cheaper than those now being achieved, switching to SpaceX could enable lower launch costs. Earlier, SpaceX placed 11 satellites (each weighing $\sim 273 \mathrm{~kg}$ ) simultaneously into an LEO implying multiple launches under a mass ceiling may also become available at potentially lower cost. If cost savings come to fruition, it will impact some architectures more than others (i.e., those for which launch costs represent a larger fraction of their overall life-cycle cost).

- Assuming assets last longer on orbit: In all other rows the model is not allowed to utilize extra on-orbit operational life as this decreases the production rate (which is technically a policy of reducing requirements). Here, however, it is assumed the disaggregated assets will experience the same extra on-orbit operational life as the GPS BAU. This reduces the production quantity of satellites (as now emergent free capability is present) and impacts the SD model, lowering efficiency. The cost presented at the points in time for this metric do not tell the whole story and the efficiency curves must also be examined to understand fully the impact of this policy.

- Note that the rows showing costs relative to the BAU where it is assumed assets last longer on orbit do not assume part commonality and reduction in launch costs over time. 
This is done to make conservative estimates and show the effects of different assumption modifications.

Table 6 2x Disaggregation summary vs. GPS BAU

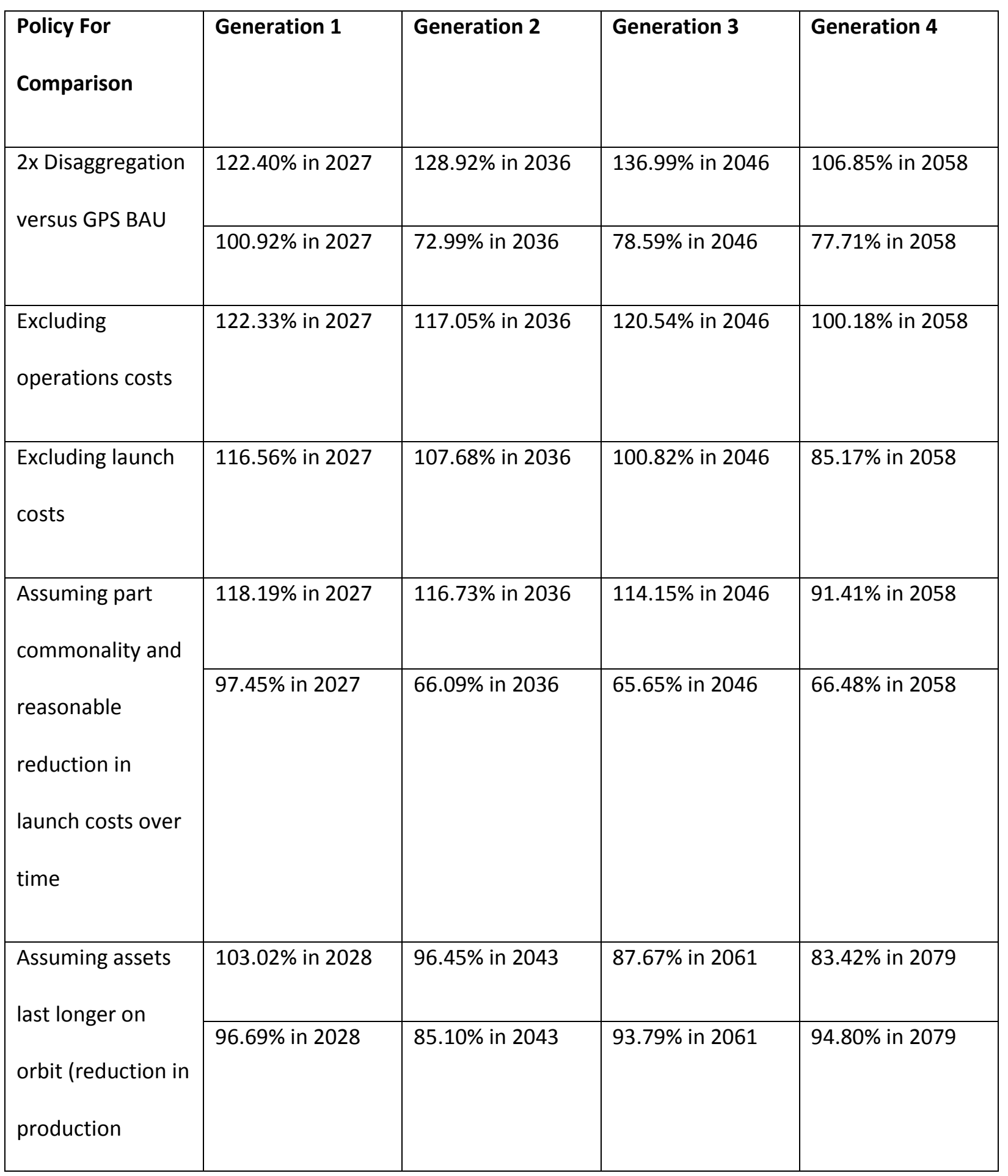




\begin{tabular}{|l|l|l|l|l|}
\hline funding) & & & & \\
& & & \\
\hline
\end{tabular}

The first row of Table 6 reveals that large cost savings are unlikely with 2x Disaggregation under basic assumptions. Even when excluding operations or launch costs, such an implementation would incur additional costs over the FYDEP. Even if part commonality is assumed and launch costs are reduced over time, FYDEP cost savings are not possible. But if these are reasonable assumptions, the amount of capability being placed on orbit and the cost per operational year of that capability on orbit may reach 67 cents on the dollar versus the GPS BAU over a 20 -year time horizon. Thus, for an estimated $14-18 \%$ increase over the FYDEP, a $2 x$ Disaggregation split of the GPS constellation is forecast to alleviate the problem of reliance on extended on-orbit design life. Furthermore, if $2 x$ Disaggregation is predicted to grant the same extended on-orbit operational life as the GPS BAU, $2 x$ Disaggregation looks even more desirable. However, (as with the GPS BAU), this leads to erosion of efficiency over time. As seen on the last row of the table, after decreasing to $85 \%$ in Generation 2, the policy gives back some of the reduction in cost to deliver capability on orbit in Generations 3 and 4. Thus, $2 x$ Disaggregation is not a policy that enables learning to take hold and establish a virtuous cycle benefiting future generations.

In Figure 26 the efficiency of the GPS BAU is displayed in blue, the efficiency of the $2 x$ Disaggregation in red, and the efficiency of the $2 x$ Disaggregation with a reduction in production funding per year as a result of capitalizing on satellites lasting longer on orbit in orange (corresponding to the last line in Table 6). A 2x Disaggregation is able to make gains in efficiency generation over generation, however, if these disaggregated satellites are expected to last longer on orbit (essentially a policy of attempting to save cost and yet maintain capability), learning effects do not take hold and efficiency drops again. Even so, this lower efficiency implementation is able to deliver capability at a savings to the FYDEP if part commonality is possible and the disaggregated satellites are able to match the aggregated systems on-orbit life. 


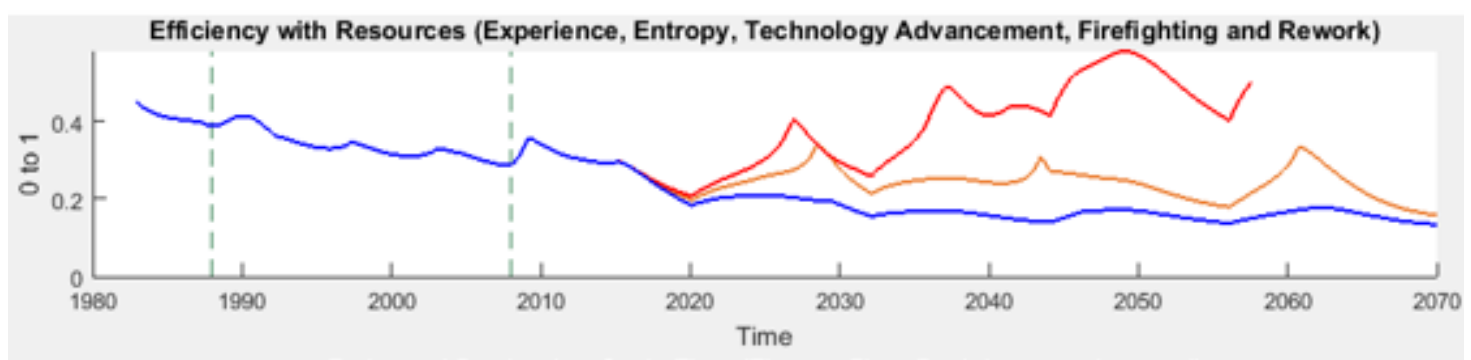

Figure 26 Efficiency for GPS BAU, 2X Disaggregation \& with reduction in production funding

Galileo-Size Disaggregation Split 3x Disaggregation

In Table 7 cost data for $3 x$ Disaggregation (also referred to as the Galileo-size implementation as each of the three satellites is about the size of a Galileo-class space craft), is displayed in the same format as for the $2 \mathrm{x}$ Disaggregation implementation. The table reveals that as with $2 \mathrm{x}$ Disaggregation no cost savings to the FYDEP are possible, and with this policy the cost may even be $50 \%$ more than the GPS BAU. While the cost per operational year on orbit is slightly lower, this is an inefficient way of paying for more capability. Yet, given the assumptions of part commonality and launch cost reductions, a different picture emerges. By the third generation, learning trumps the additional cost of disaggregation. (While not shown here, the time between generations has also shortened, assisting in technology maturation. By the third generation, this shorter development time also helps reign in cost moving forward.) If these satellites are also assumed to last longer on orbit than their design lives, then this proposal still costs more than the $2 \mathrm{X}$ split, but is cost neutral to the FYDEP.

Table 7: Galileo-Size (3x Disaggregation) Summary vs. GPS BAU

\begin{tabular}{|l|l|l|l|l|}
\hline Policy For & Generation 1 & Generation 2 & Generation 3 & Generation 4 \\
\hline 3x Disaggregation & $138.87 \%$ in 2025 & $149.92 \%$ in 2034 & $118.47 \%$ in 2046 & $110.93 \%$ in 2058 \\
versus GPS BAU & & & & \\
\cline { 2 - 5 } & $101.11 \%$ in 2025 & $76.06 \%$ in 2034 & $88.16 \%$ in 2046 & $80.68 \%$ in 2058 \\
& & & & \\
\hline
\end{tabular}




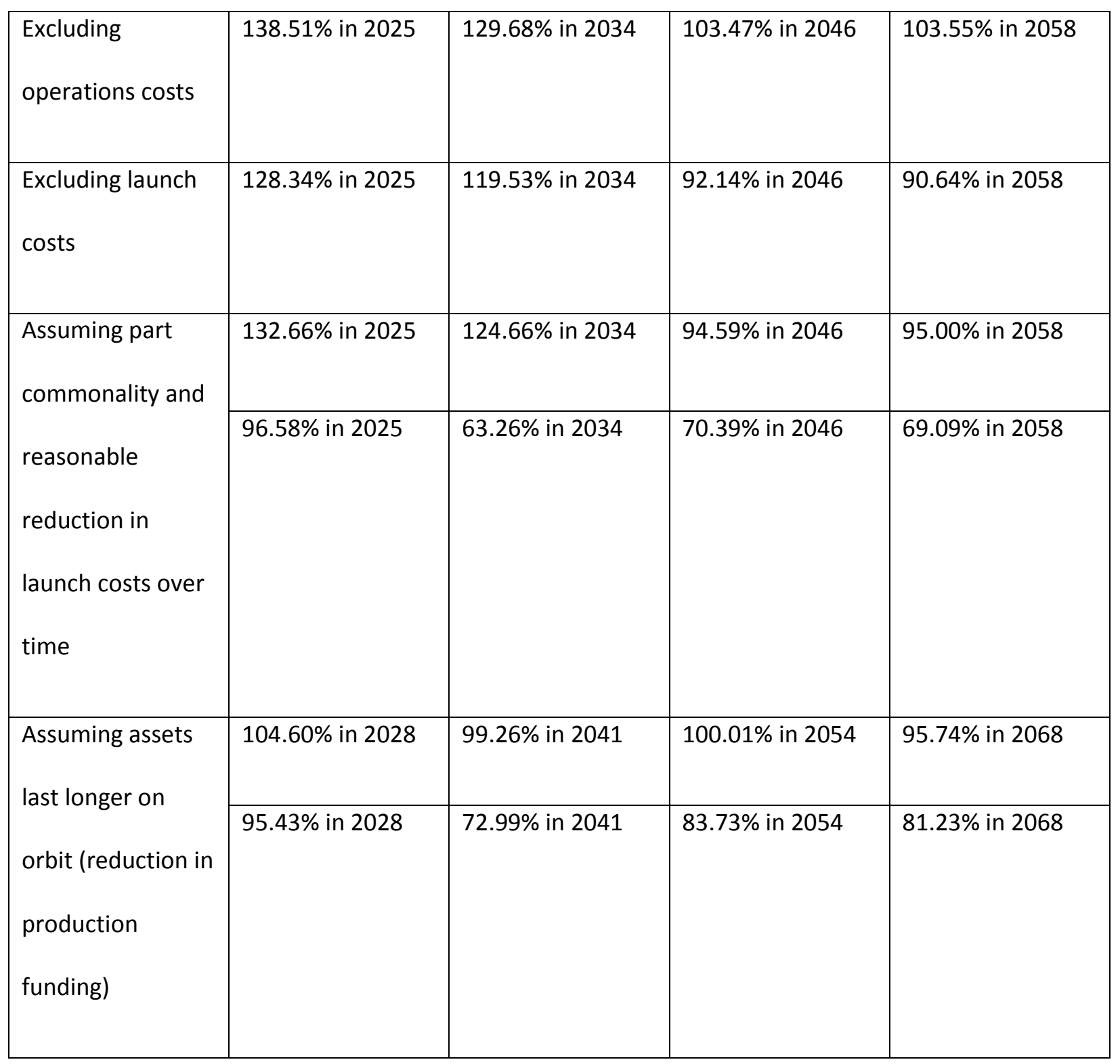

As with 2x Disaggregation, capitalizing on emergent on-orbit capability leads to degradation of efficiency as experience is decreased over the same time and Production Cycle Times are extended. Once again learning effects do not take hold, however, the shortened Production Cycle Times and greater experience generated by this policy of disaggregation do offer a cost neutral approach to the GPS BAU and delivery of $\sim 20 \%$ extra years capability on orbit. 


\section{Full Disaggregation Split 5x-6x-7x Disaggregation}

For the policy of Full Disaggregation, each satellite bus hosts only a single payload. The cost data for this policy are displayed in Table 8. Unsurprisingly, this strategy creates a massive FYDEP cost spike in the first generation, which is seen across every row in the second column (as much as $178 \%$ when compared with the GPS BAU through 2024). Over time learning does take hold and costs are more controlled, however, even under assumptions about satellites lasting longer on orbit, the additional cost of operating and launching so many more space vehicles (up to 168 satellites in the fourth generation compared to 24 with the GPS BAU) means that even large gains in production efficiency cannot overcome the increased costs from other areas of lifecycle cost. The last row of the table indicates that this radically different architecture may be available for no more than $20 \%$ increase over the GPS BAU if the same assumptions are made about extended life as is the case with the current aggregated GPS constellation. This is, at first, somewhat surprising as one might not have considered Full Disaggregation to be an efficient use of resources, however, these smaller satellites (many around $800 \mathrm{~kg}$ ), can be quickly built and lessons learned flowed back to enhance future production.

Table 8: Full Disaggregation (5x-6x-7x Disaggregation) Summary vs. GPS BAU

\begin{tabular}{|l|l|l|l|l|}
\hline Policy For & Generation 1 & Generation 2 & Generation 3 & Generation 4 \\
\hline 7x Disaggregation & $178.14 \%$ in 2024 & $146.04 \%$ in 2035 & $134.23 \%$ in 2047 & $137.80 \%$ in 2059 \\
\cline { 2 - 5 } versus GPS BAU & $110.95 \%$ in 2024 & $92.35 \%$ in 2035 & $100.93 \%$ in 2047 & $100.22 \%$ in 2059 \\
\hline Excluding & $163.81 \%$ in 2024 & $109.50 \%$ in 2035 & $101.81 \%$ in 2047 & $112.82 \%$ in 2059 \\
\hline Excrations costs & & & & \\
& & & $91.09 \%$ in 2047 & $95.36 \%$ in 2059 \\
\hline
\end{tabular}




\begin{tabular}{|c|c|c|c|c|}
\hline costs & & & & \\
\hline \multirow{2}{*}{$\begin{array}{l}\text { Assuming part } \\
\text { commonality and }\end{array}$} & $168.66 \%$ in 2024 & $126.27 \%$ in 2035 & $113.49 \%$ in 2047 & $116.02 \%$ in 2059 \\
\hline & $105.04 \%$ in 2024 & $79.84 \%$ in 2035 & $85.34 \%$ in 2047 & $84.38 \%$ in 2059 \\
\hline \multicolumn{5}{|l|}{ reduction in } \\
\hline \multicolumn{5}{|l|}{ launch costs over } \\
\hline \multicolumn{5}{|l|}{ time } \\
\hline Assuming assets & $113.47 \%$ in 2028 & $120.23 \%$ in 2039 & $112.85 \%$ in 2051 & $102.67 \%$ in 2065 \\
\hline & $102.52 \%$ in 2028 & $76.03 \%$ in 2039 & $87.48 \%$ in 2051 & $84.78 \%$ in 2065 \\
\hline \multicolumn{5}{|l|}{ orbit (reduction in } \\
\hline \multicolumn{5}{|l|}{ production } \\
\hline funding) & & & & \\
\hline
\end{tabular}

To understand why Full Disaggregation performs as well as it does, Figure 27 displays the efficiency of the GPS BAU in blue, the efficiency of the Full Disaggregation in red (corresponding to the first line in Table 8), and the efficiency of the Full Disaggregation with a reduction in production funding per year as a result of capitalizing on satellites lasting longer on orbit in orange (related to the last line in Table 8). Full Disaggregation (and the Survivable Disaggregated approach below) represents a program where learning effects take hold. The learning model as implemented should in theory, when efficiency levels reach a high enough level, enable one of two things. First, it should allow cost reductions while delivering the same capability to orbit, or second, permit delivery of more capability for the same cost. With Full Disaggregation efficiency does not decay as funding is reduced (the orange line remains close to the red). 
Unfortunately, as seen in the upper sub-row of the rows of Table 8 cost savings from a FYDEP perspective are not possible for Full Disaggregation based on current assumptions and despite this model's estimation of reduction in replication costs. Furthermore, the implementation costs more than either $2 \mathrm{x}$ or $3 \mathrm{x}$ Disaggregation policies, as significantly more mass is placed on orbit and the cost of operating such a large number of satellites eliminates any savings in production cost that result from higher efficiency.

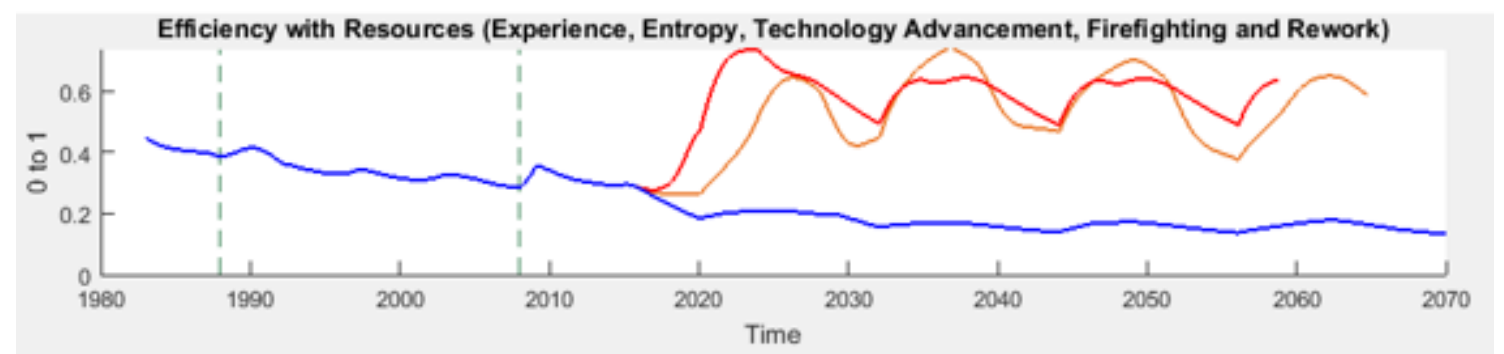

Figure 27 Efficiency for GPS BAU, Full Disaggregation \& with reduction in production funding

Survivable Disaggregation

The Survivable Disaggregated approach differs from other disaggregated approaches as it places substantially more capability on orbit. To achieve survivability, the configuration places the signal transmitters in a different configuration from the GPS BAU and other disaggregated approaches. In the first generation, one GPS satellite is split into four satellites in the following configuration:

- SV1: L1 and M1 signals

- SV2: M1 and L2 signals

- SV3: L2 and M2 signals

- SV4: M2 and L1 signals 
Future upgrades to the GPS BAU place more signals on the signal-aggregated bus; in the Survivable Disaggregated configuration each additional signal requires the addition of a new satellite to the constellation. Thus, the addition of an L5 signal in the second generation would require a satellite with the M2 and L5 signals inserted as SV4 and the new SV5 would carry the L5 and L1 signals. In the third generation, a sixth satellite would be required if an L4 signal was desired. Survivable Disaggregation would eventually result in a constellation operating six times as many satellites as the GPS BAU and would arrange signals in the following configuration: (This is given as an example, other arrangements may be more logical based upon the size of the payload, the desire to not operate all transmitters at once to save power and size, and/or other constraints outside the scope of this problem).

- SV1: L1 and M1 signals

- SV2: M1 and L2 signals

- SV3: L2 and M2 signals

- SV4: M2 and L5 signals

- SV5: L5 and L4 signals

- SV6: L4 and L1 signals

The end result of such an arrangement is that at full strength there are twice as many transmitters for each signal compared to the GPS BAU. The gain in survivability is primarily the ability for any satellite to be put out of commission with no degradation in performance (signal delivery to earth). In fact, the constellation can operate with no loss in performance with only $75 \%$ of the satellites. Even beyond a loss of $25 \%$ of the satellites, capability may degrade gracefully since the loss of additional satellites will create coverage gaps only with respect to the signals on the buses lost and because many ground receivers make use of multiple signals, even this loss may not fully degrade capability to the end user. From a constellation-wide perspective, if the same orbit and plane 
configuration as the existing GPS constellation is implemented, it is also possible that SVs could be moved within their planes over a short (multi-day) time period to cover gaps. Other advantages for this design include:

- The possibility for extensive part commonality among designs

- The production of these common parts at a rate between four and six times faster than is currently the case

- The construction of satellites which are substantially smaller than existing designs leading to faster production times (and shorter time to flow back lessons learned)

- The ability to replenish in a targeted manner as capability degrades across time

- Shorter distance between satellites enabling much smaller power consumption for crosslinks (up to four times decreased distance implying up to $16 \mathrm{x}$ less power as a consequence of the square of distance law)

- The inclusion of as many L3 payloads as desired; they can be placed on one or more satellites (The L1 and M1 as well as the L1 and L2 satellites are good candidates for easy inclusion)

- The ability to perform more risk-reduction missions as extra space is available on some of the satellites with less payload mass (e.g., flying an extra next-generation atomic clock, transmitter, flight computer or key components for a new signal generator)

The primary disadvantages are:

- Operating a larger constellation

- More rocket launches

- The SD model notes that the system can be overwhelmed initially with the increase in work if insufficient resources are supplied; a careful ramp up of production will be critical 
Table 9 displays the cost associated with fielding such a capability across time using the same conditions as for the other disaggregated approaches. A new row has also been added to the table versus the previous three, one containing the results for acquisition if a Tick-Tock policy, such as implemented by Intel (that splits R\&D into two phases: architecture upgrades and manufacturing upgrades), is employed. The Tick-Tock policy as applied to GPS splits the acquisition into two parts: one where the bus technology is updated (tick) and payloads from previous missions are flown, and a second (tock) where the payloads are upgraded and the bus remains unchanged. This policy requires more NRE to be spent as well as additional integration and test costs. This does, however, shorten the Production Cycle Time and has the potential to create cost savings versus the same acquisition without Tick-Tock.

Looking at the metrics for "Excluding Launch Costs," it can be seen that launch costs make up a larger fraction of the cost increase associated with Survivable Disaggregation than in the other disaggregated approaches. While the extra performance of Survivable Disaggregation ( $2 x$ signals and ability to operate at full capacity missing $25 \%$ of the constellation) does not come for free, it is a much more cost-effective solution than simply launching twice as many satellites of the GPS BAU class.

As with Full Disaggregation, when directly compared to the GPS BAU, Survivable Disaggregation yields a large FYDEP cost spike in the first generation (86\%). However, if this design is allowed to reap the benefits of extra emergent on-orbit design life, the increase in performance and survivability comes at a 40\% FYDEP premium to the BAU in the first generation but due to learning effects this decreases to $31 \%$ by 2058 . Moreover, as noted earlier, the calculation assuming assets last longer on orbit does not implement assumptions about reduction in launch cost or part commonality among designs. This is a worst case computation relative to the GPS BAU. 


\begin{tabular}{|c|c|c|c|c|}
\hline $\begin{array}{l}\text { Policy for } \\
\text { Comparison }\end{array}$ & Generation 1 & Generation 2 & Generation 3 & Generation 4 \\
\hline \multirow{2}{*}{$\begin{array}{l}\text { Survivable } \\
\text { Disaggregation } \\
\text { versus GPS BAU }\end{array}$} & $186.08 \%$ in 2025 & $196.06 \%$ in 2035 & $167.94 \%$ in 2047 & $162.55 \%$ in 2059 \\
\hline & $135.48 \%$ in 2025 & $105.24 \%$ in 2035 & $126.28 \%$ in 2047 & $118.21 \%$ in 2059 \\
\hline $\begin{array}{l}\text { Excluding } \\
\text { operations costs }\end{array}$ & $172.72 \%$ in 2025 & $176.75 \%$ in 2035 & $150.62 \%$ in 2047 & $154.60 \%$ in 2059 \\
\hline $\begin{array}{l}\text { Excluding launch } \\
\text { costs }\end{array}$ & $157.64 \%$ in 2025 & $135.52 \%$ in 2035 & $120.85 \%$ in 2047 & $124.30 \%$ in 2059 \\
\hline \multirow{2}{*}{$\begin{array}{l}\text { Assuming part } \\
\text { commonality and } \\
\text { reasonable } \\
\text { reduction in } \\
\text { launch costs over } \\
\text { time }\end{array}$} & $189.81 \%$ in 2025 & $178.06 \%$ in 2035 & $148.54 \%$ in 2047 & $151.20 \%$ in 2059 \\
\hline & $138.19 \%$ in 2025 & $98.63 \%$ in 2035 & $115.00 \%$ in 2047 & $109.06 \%$ in 2059 \\
\hline \multirow{2}{*}{$\begin{array}{l}\text { Assuming assets } \\
\text { last longer on } \\
\text { orbit }\end{array}$} & $141.75 \%$ in 2029 & $142.27 \%$ in 2043 & $131.1 \%$ in 2058 & $111.88 \%$ in 2076 \\
\hline & $141.75 \%$ in 2029 & $110.89 \%$ in 2043 & $126.02 \%$ in 2058 & $122.05 \%$ in 2076 \\
\hline \multirow{2}{*}{$\begin{array}{l}\text { Assuming assets } \\
\text { last longer on } \\
\text { orbit with Tick- } \\
\text { Tock policy }\end{array}$} & $142.82 \%$ in 2029 & $128.28 \%$ in 2044 & Not Computed & Not Computed \\
\hline & $139.07 \%$ in 2029 & $111.31 \%$ in 2044 & Not Computed & Not Computed \\
\hline
\end{tabular}


Looking at the efficiency of Survivable Disaggregation, Figure 28 displays the GPS BAU in blue, Survivable Disaggregated architecture in red and Survivable Disaggregated architecture while attempting to reduce costs while capitalizing on extra on-orbit design life in orange. Unlike the $2 x$ and 3x Disaggregated approaches, for Survivable Disaggregation, efficiency increases across all generations. Learning has taken hold and is enabling greater cost savings across time. This is true even when attempts are made to reduce costs by capitalizing on extra on-orbit design life. However, as the orange line is substantially below the red line, one cannot consider this a full capitalization on learning effects-efficiency is still being lost when savings cost are attempted.

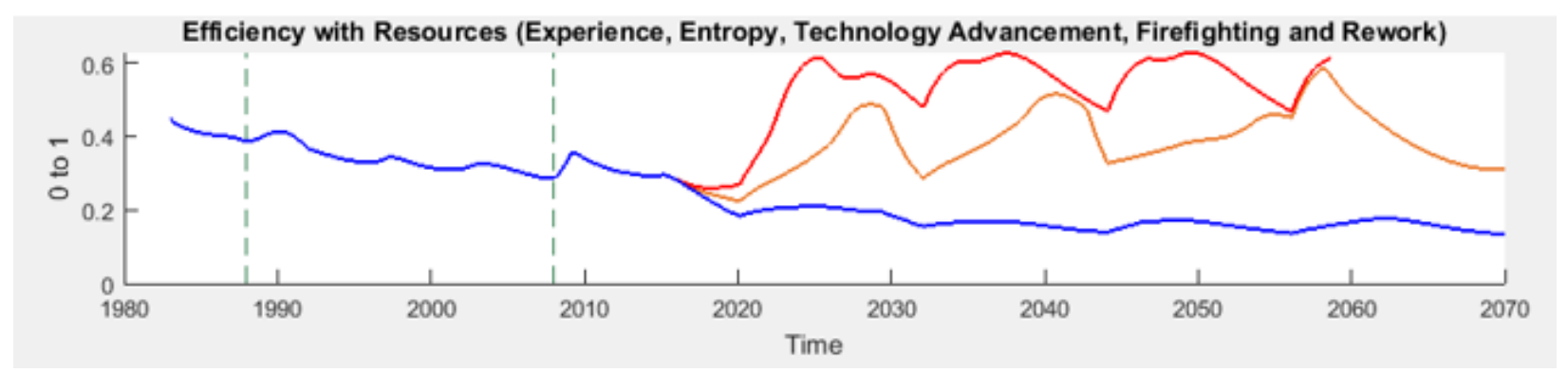

Figure 28 Efficiency for GPS BAU, Survivable Disaggregation \& with reduction in production

\section{funding}

With its shorter and more frequent bursts of NRE, Survivable Disaggregation is more efficient with a policy of Tick-Tock than without and reaches an equilibrium state more quickly (and due to the higher production quantity there are still $\sim 72$ satellite builds in each tick or tock, a sufficient number to reap the benefits of learning). This efficiency is high enough to enable learning to take hold and become robust to either requests to deliver more capability for the same cost or attempts to reduce cost while delivering the same capability without eroding the GPS production pipeline. This implementation still costs $28 \%$ more than the FYDEP from 2028 to 2044, however, this is a substantial performance increase, and policy makers may be willing to pay for such capability

The GPS BAU can now be compared against survivable disaggregated architecture in conjunction with a tick-tock policy in a future in where there is an attempt to make use of additional on-orbit life. The quantity of satellites has increased and this makes a difference for in-generation learning. These 
Survivable Disaggregated satellites while in total mass weigh nearly $2 x$ the GPS BAU, are individually small and more easily designed and manufactured. Thus, they, like the Full Disaggregation approach (with the help of the Tick-Tock policy), are able to maintain the virtuous cycle in learning. The pipeline is able to stave off erosion while being able to capture emergent extra life with on-orbit assets unlike the BAU. Also, worth noting, if extra assets are ever required this implementation is able to fulfill the need more quickly.

To directly compare this Survivable Disaggregation and Tick-Tock implementation it is instructive to look at row 9 in Table 9. From 2015 to 2029 such an implementation would require a FYDEP increase of $43 \%$. This can be regard as the premium that would be paid to have an up to date systems that is resilient to loss of GPS satellites due to some natural disaster.

\section{Conclusions}

In this work, a methodology has been developed to look at disaggregation performing over many generations to see whether the benefits of building many systems with technology refresh and an updated space industrial base outweigh the costs of building, launching and operating many more systems. This was examined using the GPS constellation as an example although the underlying ideas are much broader. GPS was used as a training set since there is a large amount of publically available data.

This work tests the policy hypothesis that (functional) disaggregation, either alone or in conjunction with other policies, is able to reduce system life cycle costs across multiple generations. The current plan to procure GPS satellites as understood by the model, was labeled the GPS BAU, and to this baseline all other policy implementations are compared. The work also examines the possibility that if cost cannot be lowered via disaggregation, perhaps more capability can be delivered for the same cost.

Key results associated with policy tests in general are as follows: 
1. Shortening design life to increase production quantity is typically a bad policy as the increased requirements to achieve extra design life costs less than the additional years of operational capability obtained.

2. The size of a block buy and the design life of satellites directly influences the time between buys. The lowest cost solutions for GPS are purchases in quantities of 12 to 18 (assuming a design life of satellites around 15 years).

3. A Tick-Tock policy can reduce the cost to acquire a year of operational capability relative to the GPS BAU

4. Smaller satellites in disaggregated approaches increase the overhead mass, require larger launch mass on orbit and incur higher launch costs. The increased gains in experience and associated efficiency in producing satellites are typically able to offset the increases in launch cost (but not all lifecycle costs).

5. The impact of Production Cycle Time or the rate at which experience can flow back and be useful for the next production cycle (represented in the SD model as the Process Cycle Time Loop) is currently more powerful (has greater impact on efficiency of building satellites) than the impact of increased Experience from the production of more units. As process cycle time drops the impact of experience becomes more valuable.

a. While increasing the amount of activity (satellites built) and thus, the amount of experience is helpful, allotting resources to shorten the time between the constructions of satellites is the first place to spend resources.

b. Current lengthy intervals between construction of satellites means that that there is little to no capitalization on experience regardless of the number of satellites built (i.e., even if you built twice as many satellites that experience would not translate into learning, unless they are also built faster). 
c. Policies of disaggregation create more experience (more satellites built) and shorten the Production Cycle Time (smaller satellites can be built faster). Together this creates the potential for capitalization on learning effects.

6. With the most successful disaggregated policies, investment (increased cost over the Future Years Budget Program (FYDEP)) occurs after disaggregation has been implemented, is required in the second generation, and required again 18 to 25 years after the policies are first enacted.

a. In no model simulation does disaggregation deliver cost savings in the first generation.

b. With disaggregation, gains in other metrics are more immediate.

i. There is a more survivable architecture and faster time to recover from catastrophic loss on orbit. (Satellites are more quickly produced and the pipeline is more responsive to increased orders.)

7. If decision makers are willing to invest in a highly survivable disaggregated architecture, increases in performance and survivability of the constellation as a whole may be available for an increase of $30 \%$ over the FYDEP by the second generation.

a. As launch costs in this architecture is $190 \%$ that of the BAU, launch costs reduction improves the cost delta.

b. If part commonality is possible among busses and additional $13 \%$ savings relative to the BAU is possible.

c. If the conservative assumption that no cost savings comes in decreased integration costs in the hosting two payloads as opposed to over six, the situation is even more favorable to this architecture. 
A general answer to the question of the economics of disaggregation is that it never makes sense economically on the first generation of satellites. However, it is possible to define a much more survivable system for a reasonable cost premium by the second generation as long as enough learning and faster production flows back into the space enterprise.

Future work would expand the capability of the SD model, for example the SD model used a single stock to measure efficiency for both across-generation and in-generation learning. In satellite production as currently practiced, production quantities are low and production times are long: each satellite is somewhat unique and a true clone is very rare. This supports combining in- and acrossgeneration learning into the same model structure. Yet the nature of learning is substantially different across generations versus within generations. When production reaches high levels, splitting this into multiple causal loops may be appropriate. While this would complicate the model, it may be worth the additional code and could simplify understanding of results with respect to first units and additional units in a generation.

The largest time requirement (by a factor of over 100) in the operation of the merged model of satellite acquisitions involves the orbital propagator (placing satellites in orbits and examining their coverage over time). The technique for the control module (constructing a level order memory tree) was written with parallelization in mind as a method of combating this computational requirement. Even so, the orbit was fixed to existing known orbits for the GPS mission area as a way of saving computational time. Future work could examine optimal ways of using coverage models in conjunction with such a technique.

\section{Acknowledgements}

The authors acknowledge useful conversations with Aerospace Corp, Profs. Sheila Widnall and David Miller in the Aeronautics and Astronautics Department at MIT and Dr. Adam Ross in the Engineering Systems Division at MIT. This work was funded in part by an Air Force Scholarship. The views 
expressed in this paper are those of the authors and do not represent the policy of the US Air Force, the US Department of Defense or the US Government.

\section{References}

[1]Satellite network robust qos-aware routing, Springer, New York, 2014.

[2]P. Anderson, L. Bartamian, Growth Trends in Communication Satellites and the Impact on Satellite System Architecture, in: American Institute of Aeronautics and Astronautics, 2008. doi:10.2514/6.2008-5440.

[3] GPS Space Segment, (2016). http://www.gps.gov/systems/gps/space/.

[4]P. La Tour, B.L. Putbrese, D. Hastings, Value-Driven Analysis of New Paradigms in Space Architectures: An llities-Based Approach, in: American Institute of Aeronautics and Astronautics, 2014. doi:10.2514/6.2014-4444.

[5]S. Loff, CubeSats, NASA. (2015). http://www.nasa.gov/mission_pages/cubesats/index.html (accessed July 22, 2016).

[6]C. Anderson, Rethinking public-private space travel, Space Policy. 29 (2013) 266-271. doi:10.1016/j.spacepol.2013.08.002.

[7]D.E. Hastings, B.L. Putbrese, P.A. La Tour, When will on-orbit servicing be part of the space enterprise?, Acta Astronautica. 127 (2016) 655-666. doi:10.1016/j.actaastro.2016.07.007.

[8] G.B. Shaw, D.W. Miller, D.E. Hastings, Development of the Quantitative Generalized Information Network Analysis Methodology for Satellite Systems, Journal of Spacecraft and Rockets. 38 (2001) 257-269. doi:10.2514/2.3679.

[9] N.H. Crisp, K. Smith, P. Hollingsworth, Launch and deployment of distributed small satellite systems, Acta Astronautica. 114 (2015) 65-78. doi:10.1016/j.actaastro.2015.04.015.

[10]J. Bloom, Eccentric orbits: the Iridium story, 2016.

[11]D. Long, B.R. Scanlon, L. Longuevergne, A.Y. Sun, D.N. Fernando, H. Save, GRACE satellite monitoring of large depletion in water storage in response to the 2011 drought in Texas: GRACE-BASED DROUGHT MONITORING, Geophysical Research Letters. 40 (2013) 3395-3401. doi:10.1002/grl.50655.

[12]S. Engelen, E. Gill, C. Verhoeven, On the reliability, availability, and throughput of satellite swarms, IEEE Transactions on Aerospace and Electronic Systems. 50 (2014) 1027-1037. doi:10.1109/TAES.2014.120711.

[13]E.M.C. Kong, D.W. Miller, Optimal spacecraft reorientation for earth orbiting clusters: applications to Techsat 21, Acta Astronautica. 53 (2003) 863-877. doi:10.1016/S00945765(02)00208-4. 
[14] Air Force Space Command, Resiliency and the Disaggregated Space Architectures, (n.d.). http://science.dodlive.mil/files/2013/08/Resiliency-and-Disaggregated-Space-Architectureswith-distro-statement.pdf.

[15] What is GOES-R, (n.d.). http://www.goes-r.gov (accessed November 23, 2016).

[16]A. Golkar, I. Lluch i Cruz, The Federated Satellite Systems paradigm: Concept and business case evaluation, Acta Astronautica. 111 (2015) 230-248. doi:10.1016/j.actaastro.2015.02.009.

[17]D. Selva, B.G. Cameron, E.F. Crawley, Rule-Based System Architecting of Earth Observing Systems: Earth Science Decadal Survey, Journal of Spacecraft and Rockets. 51 (2014) 15051521. doi:10.2514/1.A32656.

[18] J.H. Saleh, D.E. Hastings, D.J. Newman, Flexibility in system design and implications for aerospace systems, Acta Astronautica. 53 (2003) 927-944. doi:10.1016/S0094-5765(02)002412.

[19] R.E. Thompson, J.M. Colombi, J. Black, B.J. Ayres, Disaggregated Space System Concept Optimization: Model-Based Conceptual Design Methods, Systems Engineering. 18 (2015) 549567. doi:10.1002/sys.21310.

[20] R.E. Thompson, J.M. Colombi, J. Black, B.J. Ayres, Model-Based Conceptual Design Optimization Methods: Disaggregated Weather System Follow-On, Journal of Spacecraft and Rockets. 52 (2015) 1021-1037. doi:10.2514/1.A33135.

[21] M. Dwyer, D. Selva, I. del Portillo, M. Sanchez-Net, B. Cameron, Z. Szajnfarber, E. Crawley, Exploring the Trade-offs of Aggregated versus Disaggregated Architectures for Environmental Monitoring in Low-Earth Orbit, in: American Institute of Aeronautics and Astronautics, 2014. doi:10.2514/6.2014-4416.

[22] J.R. Wertz, W.J. Larson, eds., Reducing space mission cost, Microcosm Press ; Kluwer Academic Publishers, Torrance, Calif. : Dordrecht ; Boston, 1996.

[23] J.H. Saleh, Flawed metrics: Satellite cost per transponder and cost per day, IEEE Transactions on Aerospace and Electronic Systems. 44 (2008) 147-156. doi:10.1109/TAES.2008.4516995.

[24] A.M. Ross, D.E. Hastings, J.M. Warmkessel, N.P. Diller, Multi-Attribute Tradespace Exploration as Front End for Effective Space System Design, Journal of Spacecraft and Rockets. 41 (2004) 2028. doi:10.2514/1.9204.

[25] M.D. Curry, A.M. Ross, Considerations for an Extended Framework for Interactive Epoch-Era Analysis, Procedia Computer Science. 44 (2015) 454-465. doi:10.1016/j.procs.2015.03.029.

[26]J.D. Sterman, Learning in and about complex systems, System Dynamics Review. 10 (1994) 291330. doi:10.1002/sdr.4260100214.

[27] Paul A LaTour, Daniel E Hastings, Combing System Dynamics with Tradespace Exploration to explore future space architectures, in: International Astronautical Federation, Jerusalem, 2015. 
[28] Galileo Navigation Program: Fill Operational Capability, (n.d.).

https://directory.eoportal.org/web/eoportal/satellite-missions/g/galileo-foc.

[29]J.R. Wertz, D.F. Everett, J.J. Puschell, eds., Space mission engineering: the new SMAD, Microcosm Press : Sold and distributed worldwide by Microcosm Astronautics Books, Hawthorne, CA, 2011.

[30] J. B. Morrison, Implementation as Learning: An Extension to Learning Curve Theory, (2008).

[31]C.D. Jilla, D.W. Miller, Multi-Objective, Multidisciplinary Design Optimization Methodology for Distributed Satellite Systems, Journal of Spacecraft and Rockets. 41 (2004) 39-50. doi:10.2514/1.9206.

[32] Lockheed Martin Global Positioning System, (n.d.).

http://www.lockheedmartin.com/us/products/gps.html (accessed August 25, 2016).

[33]D. Chang, O. de Weck, Basic Capacity Calculation Methods and Benchmarking for MF-TDMA and MF-CDMA Communication Satellites, in: American Institute of Aeronautics and Astronautics, 2003. doi:10.2514/6.2003-2277.

[34] SpaceX launches SES-9 satellite to GEO, Space Daily. (n.d.).

http://www.spacedaily.com/reports/SpaceX_launches_SES_9_satellite_to_GEO_999.html. 